\title{
The Topology of Cultural Destinations' Accessibility: The Case of Attica, Greece
}

\author{
Areti Kranioti ${ }^{1}$, Dimitrios Tsiotas ${ }^{2, *(\mathbb{D})}$ and Serafeim Polyzos ${ }^{1}$ \\ 1 Department of Planning and Regional Development, University of Thessaly, 38334 Volos, Greece; \\ arkranioti@uth.gr (A.K.); spolyzos@uth.gr (S.P.) \\ 2 Department of Regional and Economic Development, Agricultural University of Athens, \\ 33100 Amfissa, Greece \\ * Correspondence: tsiotas@aua.gr
}

Citation: Kranioti, A.; Tsiotas, D.; Polyzos, S. The Topology of Cultural Destinations' Accessibility: The Case of Attica, Greece. Sustainability 2022, 14, 1860. https://doi.org/10.3390/ su14031860

Academic Editor: Alan Fyall

Received: 9 January 2022

Accepted: 2 February 2022

Published: 6 February 2022

Publisher's Note: MDPI stays neutral with regard to jurisdictional claims in published maps and institutional affiliations.

Copyright: () 2022 by the authors Licensee MDPI, Basel, Switzerland. This article is an open access article distributed under the terms and conditions of the Creative Commons Attribution (CC BY) license (https:// creativecommons.org/licenses/by/ $4.0 /)$.

\begin{abstract}
This paper studies the cultural landscape in the prefecture of Attica, Greece, within the context of network science. Tourism is a global and complex phenomenon with a considerable effect on space. Among its diverse forms, cultural tourism stands out, since people often travel to explore different cultures and civilizations. The engagement of different stakeholders in the establishment of policies in regard to tourism development and its relation to cultural heritage demonstrates the need for sustainable spatial planning in cultural tourism areas. According to network science, a modern discipline already contributing to the research in tourism geography, tourism destinations can configure complex spatial networks and be studied in terms of network analysis and statistical mechanics. This paper models the accessibility network of cultural destinations in Attica (ACDN), the capital region of Greece, into a graph, which provides an excellent case study of multilayer network modeling, as is equipped with high quality transportation, accommodation, and cultural infrastructures. The ACDN nodes represent museums and archaeological sites and its edges express possible connections between nodes by different means of transport (car, pedestrian, and public transport). The analysis aims to evaluate the layer's capacity in the service of the accessibility of the cultural destination tourism market in Greece and reveals two major communities in the structure of ACDN, an urban core and a peripheral, configured by spatial constraints and distinct transportation mode functionality, along with uncovering deficiencies in the public transportation setting of the cultural tourism market of Attica. Overall, this study highlights the requirement for spatial planning and tourism management to be aware of geographical, topological, and functional features of a cultural tourism market and promotes the symbiotic relationship of sustainable tourism development and network science.
\end{abstract}

Keywords: cultural tourism market; accessibility network; tourist mobility; network analysis; community detection

\section{Introduction}

Tourism is a global, social, cultural, and economic phenomenon, with a high impact on space mainly due to the development of supporting activities, such as accommodation and leisure, and tourist movements and flows [1-5]. When it involves culture, tourism specializes to a form of cultural tourism, which suggests a sustainable drive of development, resulting in economic growth for local communities while contributing to cultural preservation and environmental protection $[1,6]$. In the current literature, cultural tourism enjoys multidisciplinary research, ranging from qualitative to empirical studies [6-26]. In the last decade, the newly established discipline of network science [27-30] has contributed in the study of tourism destinations with the use of network paradigm [12-21]. However, network analysis currently appears to emphasize more on the social actors' than on accessibility configuration of the cultural tourism market. Towards the demand of studying tourism 
geography by using the network paradigm [29], this paper provides an empirical study on the spatial aspect of cultural tourism by examining the level at which the geography and network topology of cultural tourism destinations of Attica (Athens), Greece, serve tourism demands and promote tourism. Athens is a Greek city with remarkable cultural capital [4], where its historic center acts as a "pole" for visitors, while other archaeological sites and museums in the metropolitan area remain unknown to the cultural tourism market [7]. Attica is Greece's highest inhabited region, including the country's capital city, Athens [4]. The area is known worldwide for its archaeological sites, monuments, and museums - testimonies of a rich historical past - that attract a significant number of tourists annually. In 2019, the Acropolis Museum had roughly 1.8 million visitors, Acropolis hill had 3.5 million, and the National Archaeological Museum had more than half a million, respectively [31]. One major problem in the cultural tourism market of Attica is its numerous and geographically scattered destinations [4], making the optimal management of these various cultural tourism areas, both at the microeconomic (from the visitors' side, in terms of choosing the best possible set of destinations within certain holidays time) and macroeconomic level (from the tourism management and policy perspective, in terms of optimizing the accessibility and development of transport infrastructures to support and money expensive) a challenge [8]. Since cultural capital is a sunk cost, inflexible, and impossible to geographically rearrange, it is of great importance for tourism management to evaluate its accessibility given that spatial constraints are inevitably applicable [8]. Therefore, the study of cultural tourism in a destination, in terms of mobility and connectivity, is a promising approach that has a lot to offer, especially when it comes to the demand of sustainable tourism development. Within this context, this study conceives the cultural landscape of Attica as a complex network and examines the connectivity of its destinations, the topological relevance between different transport modes, and the contribution of each transport mode to the overall network topology. The analysis aims to provide insights into the level at which the geography of tourism destinations is related with the attractiveness of a cultural tourism market and whether accessibility management of these high sunk cost destinations can optimally occur. The remainder of this paper is structured as follows: Section 2 provides a literature review; Section 3 presents the methodological framework and the data of the study; Section 4 shows the results of the analysis and discusses them within the context of tourism management and policy; and finally, in Section 5, conclusions are given.

\section{Literature Review}

Tourism and culture have a strong connection, as culture has been one of the main motives for travel globally [7]. This relation among the two concepts has resulted in a specific type of tourism, known as cultural tourism. In 2017, the United Nations World Tourism Organization (UNWTO) [1] defined cultural tourism as "a type of tourism activity in which the visitor's essential motivation is to learn, discover, experience and consume the tangible and intangible cultural attractions/products in a tourism destination". According to this definition, a tourist can experience different aspects of culture in a destination. As a type of tourism, cultural tourism is one of the most upcoming trends worldwide in tourism, and its symbiotic relationship with sustainability plays a crucial role towards this direction $[3,6,9]$. Although cultural tourism has been criticized for its negative effects on local communities to the extent that it is related to massification (carrying capacity problems), the positive effects of cultural tourism prevail, amongst which include the following [1,4,9]: (i) the strengthening of local culture, (ii) the development of a unique branding image for each region, (iii) environment and culture protection, which is a definitive property of cultural tourism, and (iv) the potential to expand tourism seasonality, provided that cultural tourism is unrelated to the $3 \mathrm{~S}$ mass tourism model. The broad nature of cultural tourism has attracted interdisciplinary research, such as sociology, economics, anthropology, and psychology, and it is studied not only from a supply-demand perspective but also through theoretical and practical approaches; in particular, as a social phenomenon, it has been 
studied under different disciplines and methodological approaches since the 1980s [6,10]. In his review article of [10], the author lists the research streams encountered so far in the cultural tourism literature. In terms of cultural consumption and related motivations, the focus is on qualitative studies and correspondence analysis to define the profiles of cultural tourists and consumer behavior. On the contrary, the economic aspect of cultural tourism has been studied in the context of time-series analysis and forecasting models. The aim of such papers is to better understand the need for supporting facilities, such as accommodation, transportation, and gastronomy, helping destinations to develop and plan accordingly. The last research stream, according to [10], includes empirical studies in cultural destinations that investigate the links between cultural capital and tourism in a manner to achieve sustainability of heritage. During the last decade, there has been a shift in the field of tourism research, where a significant number of papers use network analysis as a methodological context. The author of [10] observes that network science, as a methodology, can identify synergies and links among different actors in the cultural tourism market.

In the relevant literature [10-13], one subject of particular interest for network analysis in tourism destinations is the role of different stakeholders and sectors involved in the tourism industry and the way these actors interact and collaborate. The paper of [11], constructed a network model of government sectors involved in the post-disaster management of Jiuzhaigou National Park, where edges represented the relations between sectors, and evaluated the way nodes interacted to address the problems caused by the disaster. Various network measures (density, average distance, centrality, and clique) were calculated to identify the collaboration structure among the stakeholders. The paper of [12] applied network analysis to hospitality firms and official stakeholders in Molise region, Italy, to examine the collaboration possibilities among officials and service providers. The analysis is built on degree centrality to evaluate the firms' preference to collaborate with the region's officials, on closeness centrality to indicate the preferable stakeholder, and uses other centrality indicators to describe the marketing activity. The authors of [13] studied tourism services distribution channels in Hanoi, Vietnam, using network measures (density, clustering coefficient, and closeness centrality) and organizational risk analysis to study the relations between travel agencies and tour operators based on their online presence and identified patterns of the information flows in the network. On the other hand, other studies [14-21] are also interested in tourists' perception of a destination. Toward this direction, relevant papers analyze the nature of the linkages of nodes in tourist attraction networks of travel destinations, with most of the cases studied so far located in Asia. The author of [14] used network analysis to study the tourism characteristics of 16 destinations in Nantou, Taiwan, and identified the structural patterns among these destinations by analyzing the degree, betweenness, and closeness centrality of each node, providing insights into tourist facilities needed in each destination based on their characteristics. Next, the work of [15] combined GIS, social network analysis (mapping the attractions' degree and eigenvector centrality with GIS data), and spatial statistics to study tourist attraction networks in Seoul, South Korea, and, in particular, to recognize spatial distribution patterns among 29 tourist attractions and associate them with the length of stay in Seoul. The paper of [16] modeled the tourist attraction network in Shanghai, China, into a complex network constructed on travel notes and official news data and compared this network model with the one suggested by the government's attraction network. By using network analysis and statistical mechanics, the study highlighted the interdependence among the attractions and the inconsistencies between the official attraction network and the one formed by the traveler's perspectives. In the same direction, the authors of [17] examined the mechanisms resulting in the development of an attraction network in Xinjiang, China. By comparing the locational closeness of the attractions, their operation time, the volume of visitors, and the degree centrality of the attractions, they specified the relations formed among the nodes of the attraction network. In a recent paper, the authors of [18] evaluated the accessibility of suburban attractions in Beijing, China. Building on GIS network analysis, they investigated 
the spatial differences in reaching the attractions by car, public transport, and bike and proposed strategies in tourism development in Beijing suburban area. The authors of [19] studied a network of historical ensembles in Extremadura, Spain, to contribute to tourism planning in the context of creating thematic (expressing a peculiar kind of resource) tourist itineraries. The study showed the potential of generating tourist itineraries as a first step in the design of tourist products and highlighted the utility of network analysis as a management tool of a coherent and efficient tourist policy. The paper of [20] examined recurrent spatial patterns of package holidays in mainland Portugal by applying a cluster analysis to a network of cultural attractions weighted by the number of overnight stays per municipality. The analysis showed that organized tourist programs, based on cultural heritage break, reduce to some extent the spatial asymmetry of Portuguese tourism model, which was historically concentrated around the cultural attractions, promoting a decentralized operational plan in the topology of cultural network from the strategic gateways (Lisbon and Oporto) to peripheral municipalities. Finally, at the global geographical scale, the authors of [21] studied the topology of the Global outbound and inbound Tourism Network (GTN), consisting of 214 countries worldwide and found that the network is ruled by a good hierarchical configuration (as described by the scale-free property), large transitivity, high-degree centralization, and a clustered structure determined by geographical along with trade and cultural factors. The authors also observed that the network has small-world characteristics and a high degree of geographic homophily (with more links within than between continents), interpreting that GTN emerges from superimposed local processes in which tourism flows between countries are determined from multiple independent individual decisions made at the local level.

As is evident, network analysis is evolving in the research of cultural tourism and currently case studies enjoying such an approach are places with a considerable cultural capital. Being a region of notable cultural capital, Attica, Greece [4], can provide an excellent case study to apply network analysis and examining the symbiotic relation deeper between accessibility and cultural tourism attractiveness. However, current tourism research in Attica mainly focuses on two research directions [22-26]. In the first, a significant number of papers focus on the Olympic Games 2004 in Athens and the following economic crisis and the aftermath impact on the city's image and its tourism development. Tourism in Athens and Attica, in general, was evolving after the Olympic Games in 2004, but the development came at stake with the economic crisis [22-24]. The work of [22] built on semi-structured interviews with officials to investigate Athens' potential to be a cultural destination in the post-Olympic era. A major finding focused on the constraints involving the cost of managing all existing cultural areas and venues and the lack of related policies. In the work of [23], the authors examined the evolution of Athens' image from the visitors' perception in the years of economic recession. Based on data extracted from the TripAdvisor website, the authors observed that Athens shaped a positive image through the years, despite the economic crisis. However, as the authors of [24] observed, Attica was one of the prefectures that were seriously affected by the economic crisis, as shown in the reduction in tourist stays in the area since 2008. The second research direction deals with further tourist development in the prefecture, where an interesting potential is found in many areas and the focus lies on the coastal areas in Attica and the area's given cultural capital, provided that the cultural capital of Greece, and especially the one of Attica, is a strong comparative advantage against the constantly growing competition among tourist destinations in the Mediterranean [7]. The authors of [25] recognize the cultural resources in the Attica region that act as a tourist pole, underlining the strong potential of the area's coastal municipalities and trying to address issues related to tourist development. Following the same research direction, the author of [26] suggests an alternative tourism model for Greece that diversifies the traditional Greek tourist product with the promotion of the country's industrial heritage. The so-called Lavreotiki area, in Attica, is highlighted as a case study and the historic industrial complex of the French Mines Company in Lavrion is suggested as an alternative touristic destination to ensure the preservation of the complex while spreading tourist 
flows away from the crowded Athens historical center. In any case, Attica has a strong potential to develop as a cultural destination due to its unique cultural asset, archaeological sites, historical monuments, and museums and its high quality tourist infrastructure [7]. However, as the previous studies show, the problem is the cost of managing cultural areas in Athens and supporting them with tourism-related infrastructure. Towards this direction, this paper models the cultural landscape of Attica into a complex network, aiming to provide insights into the level at which the geography of tourism destinations is related with the attractiveness of this cultural tourism market, within the context of optimally managing the accessibility of destinations, as a major drive towards sustainability in tourism and regional development.

\section{Methodology and Data}

This paper models cultural tourism destinations of Attica prefecture, Greece, into a multilayer network (Attica's Cultural Destinations Network-ACDN) consisting of three layers of different accessibility modes and applies complex network and empirical (statistical) analysis to examine its topology compared to its geographical configuration. The methodological framework comprises five steps: The first one regards graph modeling [28] ACDN; the second step includes network analysis [32,33], which is applied between the network layers and the aggregate network aiming to detect topological similarities and differences and to compare the accessibility potential of ACDN between its transportation modes; the third step deals with community detection [34], aiming to separate the multilayer ACDN into communities (under the modularity optimization criterion) with the best possible interior connectivity and, therefore, to detect areas of relevance based on accessibility in Attica's cultural tourism market; the fourth step regards an empirical analysis examining the topology and some major tourism attributes of the communities emerged by the modularity optimization algorithm; finally, in the fourth step, conclusions are formulated within the context of tourism management and geography. The particular steps of the methodological framework are described in more detail in the following paragraphs.

\subsection{Graph Modelling and Data}

The cultural landscape of Attica's (Greece) mainland (Figure 1) is modeled to a multilayer weighted undirected graph $M(G, C=\varnothing)$ consisting of three (3) layers $G=\left\{G_{p}\right\}=$ $\left\{V_{p}, E_{p}, p=1, \ldots, 3\right\}$ without interlayer connections $C=\left\{E_{i j} \subseteq V_{i} \times V_{j}\right\}=\varnothing$ [35], where $V$ expresses the node set and $E$ the edge (links) set. The multilayer ACDN is a multiplex graph, where the same number of nodes applies to all layers $\left|V_{1}\right|=\left|V_{2}\right|=\left|V_{3}\right|=n=31$. Nodes in multiple ACDNs express 31 cultural tourism destinations in Attica (see Table A1 in the Appendix A) and, in particular, the museums and archeological sites extracted from the list of the Greek Ministry of Culture, in Attica, as it is shown in Figure 2. The first layer $G_{1}\left(n_{1}=n=31, m_{1}=91\right)$ represents the car accessibility layer of ACDN, where edges express the potential to reach a destination (node) by car. This layer is a disconnected (not all nodes are accessible through a path in this layer), undirected, and a weighted spatial network, where edge weights express kilometric and time distances extracted from the [36] official website. For the sake of simplicity, we denote this layer as $G_{1} \equiv \operatorname{ACDN}_{(\mathrm{C})}$, where reference index " $\mathrm{C}$ " stands for "car."

The second layer $G_{2}(31,94)$ represents the pedestrian accessibility layer of the ACDN, where edges express the potential to reach a destination (node) on foot. This layer is a connected, undirected, and weighted spatial network, with weights expressing kilometric and time distances. Let us denote this layer as $G_{2} \equiv \operatorname{ACDN}_{(\mathrm{P})}$, where reference index "P" stands for "pedestrian." The third layer $G_{3}(31,26)$ represents the public transportation accessibility layer of the ACDN, where edges express the potential to reach a destination (node) via a public transportation mode (bus, railway, tram, etc.). This layer is also a disconnected, undirected, and weighted spatial network, with weights expressing kilometric and time distances. For the sake of simplicity, we denote this layer as $G_{3} \equiv \operatorname{ACDN}_{(\mathrm{T})}$, where reference index " $\mathrm{T}$ " stands for "(public) transportation." 


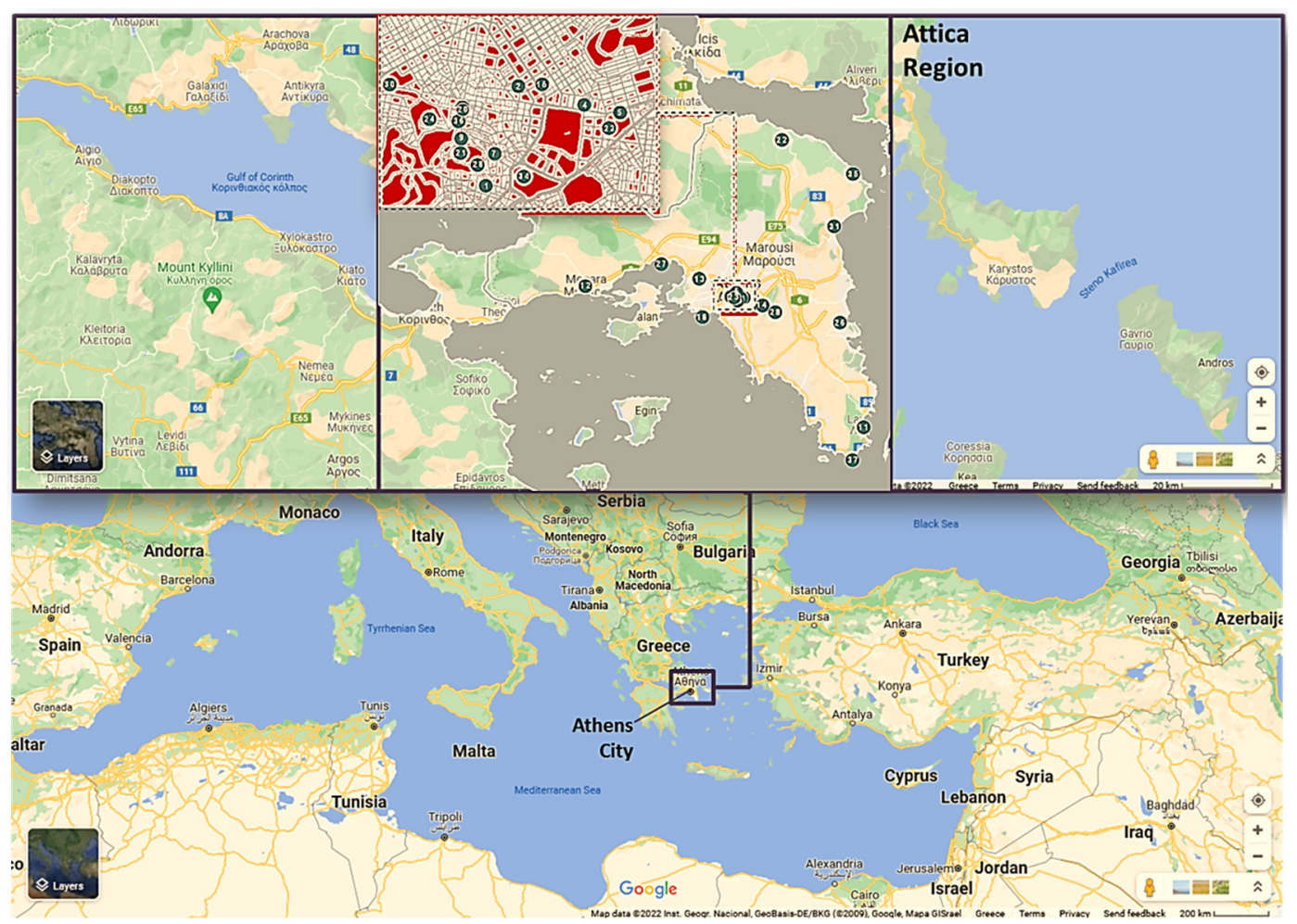

Figure 1. The location of the ACDN nodes in Attica, Greece (own elaboration based on Google Maps).
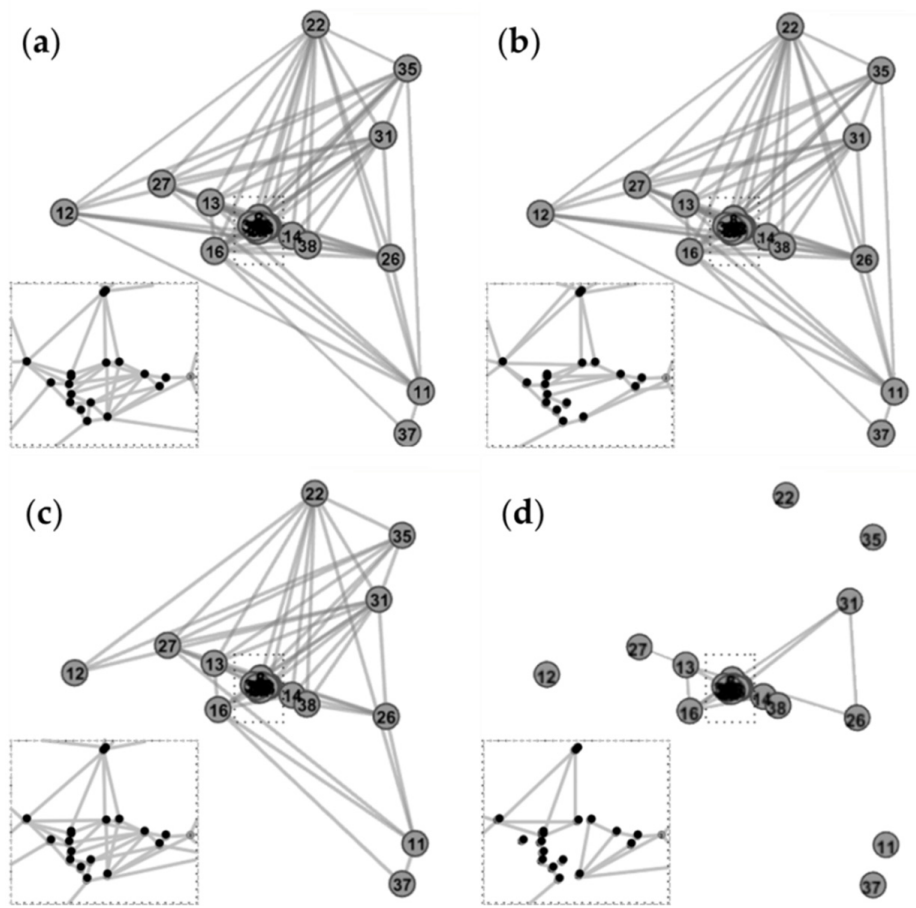

Figure 2. Geo-referenced (Web Mercator) graph layouts of the cultural network od Attica in the L-space representation, where $(\mathbf{a})$ is the aggregate $\left(\mathrm{ACDN}_{(\mathrm{A})}\right)$; $(\mathbf{b})$ is the $\operatorname{car}\left(\mathrm{ACDN}_{(\mathrm{C})}\right)$; (c) is the pedestrian $\left(\mathrm{ACDN}_{(\mathrm{P})}\right)$; and $(\mathbf{d})$ is the public transportation $\left(\mathrm{ACDN}_{(\mathrm{T})}\right)$ layer.

Finally, we consider in the analysis the aggregate ACDN layer, computed by the set theoretic union of the three available layers $A C D N_{(A)}=\underset{i=C, P, T}{\cup} A C D N_{(i)}$, where reference index " $\mathrm{A}$ " stands for "aggregate." This layer $G_{0}(31,116) \equiv A C D N_{(A)}$ represents the overall 
accessibility layer of the ACDN, where edges express the potential to reach a destination via any available transportation mode. The aggregate layer is connected, undirected, and weighted spatial network, with weights expressing kilometric and time distances. Nodes in the multiplex ACDN are geo-referenced using the (Google) Web Mercator map projection, and they are positioned in the map according to their coordinates (LAT, LONG) extracted from [36]. ACDN and all its layers are constructed in the L-space [28] representation, where nodes $(\mathrm{V})$, expressing the museums and the archeological sites of Attica, are connected by edges (E) if they are consecutive stops on a given route (Figure 2). In the topological layout, edges are drawn as linear segments and their real geographical distances are assigned as weights. All distance and time distance data were extracted from [36].

\subsection{Network Analysis}

After multilayer graph modeling, we study the topology of the multilayer ACDN by using a set of network measures shown in Table 1. Each measure captures certain aspect(s) of network topology (connectivity, intermediacy, path length, clustering, etc.), and a common consideration can provide a multifaceted approximation of the overall network topology [37]. Measures referring to the entire $G$ network are considered as global, whereas those referring to network components (nodes and edges) are considered as local [32].

Table 1. Measures of network topology used for the analysis of the ACDN.

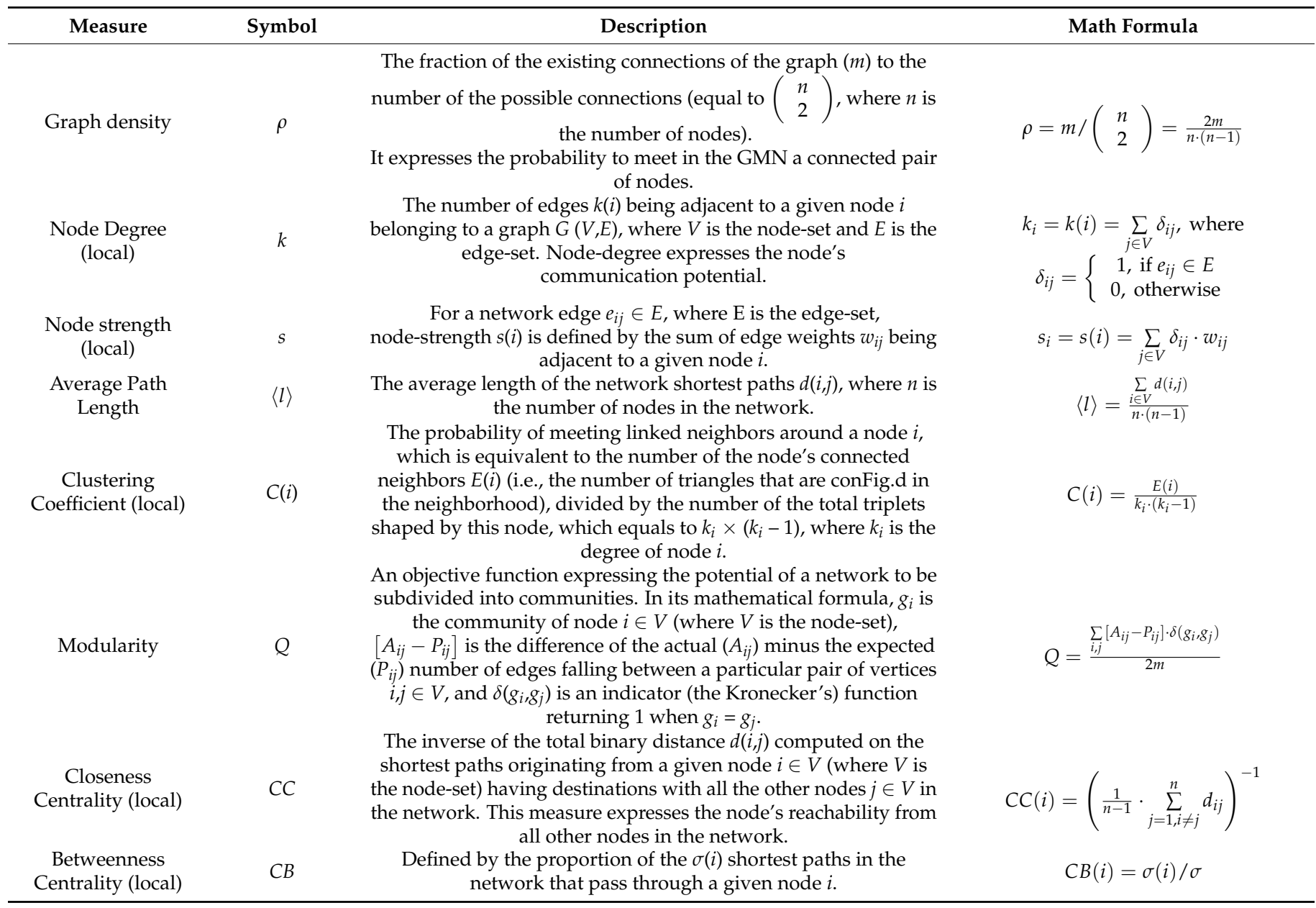

Sources: Own elaboration based on [32-34].

Direct comparisons of network measures between layers are possible only for those measured by unit-free or dimensionless numbers, such as node degree, clustering coefficient, binary average path length and network diameter, graph density, and modularity $[32,33,35,38]$. On the other hand, weighted measures, such as average path length, edge 
length, weighted network diameter, etc. [28,33], are not directly comparable unless they submit to normalization or rescaling $[39,40]$. Moreover, network connectivity matters for the sake of comparisons between networks (or layers). For instance, network distance cannot be computed between disconnected nodes; thus, it should be conventionally defined. Moreover, nodes of the same network measure that are included in components of different sizes may not have the same importance for the system represented by the network. In general, this problem is known in network science as "insufficient connectivity" and several repairing methods have been developed to overcome certain restrictions, mainly depending on modeling and the purpose of the research. For this study, we use the local restriction method-LRM [32] — which is the simplest between the available methods because it converts measures from local (within their connected components) to global (referring to the aggregate network) without any modifications (as they are). This is an accepted approach for ACDN, first, because in terms of definition, the nodes represent museums and archaeological sites and, therefore, distinguish their importance as impractical; and, secondly, in methodological terms, empirical analysis builds on inferential computations that are expected to counterbalance any bias caused by insufficient connectivity.

For pattern recognition, the analysis builds on a double approach; first, it examines the type of degree distributions $p(k)$ to obtain insights into the network's structure and functionality, along with the growth mechanism of networks [28,37,41]. In general, the degree distribution $p(k)$ is defined by the frequency distribution $\left(k_{i}, n\left(k_{i}\right)\right)$ of the unique values $k_{i}$ of the node degrees in the network and is expressed by the following relation [41]:

$$
p(k)=\left(k_{i}, n\left(k_{i}\right)\right)
$$

where $n\left(k_{i}\right)$ is the node frequency (number of nodes) of degree $k$. When divided by the total number of nodes $n\left(k_{i}\right) / n$, the degree distribution can become a probability distribution $[28,41]$, thus expressing the probability to meet a node of degree $k_{i}$ in the network. Theoretical and empirical research in complex network $[28,37,38,41,42]$ has shown that the type of degree distribution can be representative of network topology. Within this context, comparisons of the ACDN's degree distributions both between layers and in reference to theoretic (null) models can provide insights into the similarities and the level of hierarchies in the structure of ACDN and its layers. A second approach on pattern recognition builds on the examination of the sparsity (spy) plot $[37,42]$ of the graph's adjacency matrix. A spy plot is generally a dot representation of a matrix displaying nonzero elements with dots. Within this context, the spy plots can be insightful for pattern recognition in graphs because they illustrate graph connectivity in a matrix space, where, for instance (for certain node ordering), patterns of concentration along the main diagonal may represent lattice-like connectivity and are asymmetrically scattered throughout the matrix, expressing random network structures [37,42].

\subsection{Community Detection Based on Modularity Optimization}

At the third step of the methodological framework, we apply community detection analysis to the ACDN by using the modularity optimization algorithm proposed by [34]. The modularity optimization is a greedy algorithm dividing a graph into communities according to their node connectivity, requiring maximizing intra-community (within the communities) connectivity and, consequently, minimizing the (inter-community (between the communities) connectivity, as expressed in the following criterion relation:

$$
\operatorname{maximize}\left[Q \propto\left(m_{\text {withincommunities }}-m_{\text {betweencommunities }}\right)\right]
$$

where $Q$ is the modularity function and $m$ is the number of links. Modularity is generally an objective function defined by the sum of actual minus, the expected number of edges falling in a community, as shown in Table 1. The modularity optimization algorithm applies at two stages [34]; at the first stage, each node in the graph is registered in a separate community. Next, the nodes are step-by-step swept and placed into collective communities provided 
that a collection increases the gain in the weighted modularity function $\left(Q_{w}\right)$ of the initial graph. At the second stage, the collective communities are replaced by "super-nodes" and the procedure is repeated until the modularity function converges (cannot increase any more). Nodes included in the same community are relevant in terms of connectivity than those included in different communities, in the context of the functional attribute expressed by a connection. In this paper, we apply the community detection algorithm of [43], which is available in the open source software of [44].

\subsection{Empirical Analysis}

At the fourth step of the methodological framework, we apply empirical analysis aiming to detect distinguishable attributes in the communities generated at the community detection analysis. This approach assumes that connectivity emerges as a result of functional interaction and spatial proximity in spatial communication systems; thus, it applies further analysis to detect the underlying topological-geographical and socioeconomic forces generating the ACDN communities, provided that is already known that members of the same community are relevant with each other but communities are distinguishable and, thus, different in terms of network connectivity. Within this context, empirical analysis applies both to a set of topological variables (collected from the available local node measured of Table 1) and to the set of tourism-defined socioeconomic attributes shown in Table 2.

Table 2. Tourism-defined socioeconomic attributes considered as node variables in the empirical analysis of ACDN.

\begin{tabular}{ccc}
\hline Attribute & Description & Data Source \\
\hline Visitors & Node's total visitors in 2019 & {$[31]$} \\
Revenue & Node's total revenues in 2019 $(€)$ & {$[31]$} \\
Establishment Date & The year that each site was & proclaimed to museum \\
Node's admission fee during & summer 2019 & Node's official website \\
Admission Fees & Number of neighbor hotels & \\
& registered in & \\
Hotels & TripAdvisor database within a 2 km & distance range \\
& [45] & \\
\hline *. Own elaboration based on Museum official websites on the Catalogue of Listed Archaeological Sites.
\end{tabular}

The empirical analysis builds on the idea of the independent-samples t-test and is used to compare the means (in 0.05 significance level) between two discrete groups defined from the same variable $X$ by using either an arithmetic (i.e., cutting point) or categorical grouping criterion. A visualization of this test can be achieved by using error bars of 95\% confidence intervals (CIs) for the means [39,40]. In the context of ACDN, available topological and tourism-defined socioeconomic variables are separated into groups according to their membership in community detection, and afterward, 95\% CI error bars were computed to examine any differences between the attributes describing the modularity communities. When CIs overlay, the mean values between communities are statistically the same, whereas when they do not overlay, the mean values are considered as statistically different, interpreting that the communities' performance differs in terms of the measured mean value attribute.

\section{Results}

\subsection{Network Analysis}

After modeling the multilayer ACDN, we apply a network analysis consisting of calculation of network statistics (measures of network topology) and pattern recognition based on degree distributions and sparsity (spy) plot examination. At first, the results of network statistics are shown in Table 3, where rows refer to certain network measures and columns to the available ACDN layers: the aggregate $\left(\operatorname{ACDN}_{(\mathrm{A})}\right)$, the car $\left(\mathrm{ACDN}_{(\mathrm{C})}\right)$, the 
pedestrian $\left(\mathrm{ACDN}_{(\mathrm{P})}\right)$, and the public transportation $\left(\mathrm{ACDN}_{(\mathrm{T})}\right)$ layer. As observed, the aggregate layer $\mathrm{ACDN}_{(\mathrm{A})}$ has a greater number of connections than each layer separately, implying (in a first reading) that layers cooperate in the formation of the total ACDN. However, the inequality $116 \neq 91+94+26$ implies that the contribution of each layer to the configuration of the aggregate $\mathrm{ACDN}$ is not a summative process due to the graph theoretical union defining the following relation:

$$
E_{(A)}=\underset{i=C, P, T}{\bigcup} E_{(i)} \Rightarrow\left|E_{(A)}\right|=\underset{i=C, P, T}{\bigcup}\left|E_{(i)}\right| \Leftrightarrow 116=91 \cup 94 \cup 26
$$

according to which some edges coincide and form double links. Within this context, the pedestrian layer can be considered as more relevant to the aggregate layer $\mathrm{ACDN}_{(\mathrm{A})}$ because it incorporates $91 / 116=78.5 \%$ of the total connections configuring ACDN. On the contrary, public transportation $\mathrm{ACDN}_{(\mathrm{T})}$ can be considered as more distant to the structure of the $\mathrm{ACDN}_{(\mathrm{A})}$ because it only includes $22.4 \%$ of the ACDN's links. In terms of number of components, graph density, average degree, network diameter, and average path length, layer $\mathrm{ACDN}_{(\mathrm{P})}$ is the most relevant to the aggregate network, illustrating the importance of the pedestrian mode in the structure of cultural tourism destinations market's accessibility (where no special infrastructure is required to access the destinations). However, in terms of maximum degree and average strength (weighted degree), we can observe that the car layer is closer to the aggregate layer's configuration. As far as maximum degree is concerned, this result highlights the importance of the private transportation mode in the connectivity of the cultural tourism destinations market. As far as weighted average degree is concerned, which provides information about the layer's functionality in the network, these results illustrate that the car appears to be a better determinant of $(i)$ the average accessibility time (time-weighted degree) and (ii) the proximity of the total network, implying that the private transportation prevails in the functionality (time and geographical accessibility) of the ACDN.

Table 3. Network measures computed for the analysis of ACDN.

\begin{tabular}{ccccc}
\hline & $\mathbf{A C D N}_{(\mathbf{A})}$ & $\mathbf{A C D N}_{(\mathbf{C})}$ & $\mathbf{A C D N}_{(\mathbf{P})}$ & $\mathbf{A C D N}_{(\mathbf{T})}$ \\
\hline Nodes $(\mathrm{G})$ & 31 & & $\mathbf{3 1}$ & \\
Edges $(\mathrm{E})$ & 116 & 91 & $\mathbf{9 4}$ & 26 \\
Components & 1 & $\mathbf{1}$ & $\mathbf{1}$ & 19 \\
Graph density $(\rho)$ & 0.249 & 0.196 & $\mathbf{0 . 2 0 2}$ & 0.056 \\
Maximum degree $\left(\mathrm{k}_{\max }\right)$ & 14 & $\mathbf{1 3}$ & 11 & 5 \\
Average degree $(\langle k\rangle)$ & 7.784 & 5.871 & $\mathbf{6 . 0 6 5}$ & 1.677 \\
Average time - & 269.61 & $\mathbf{1 8 1 . 8 7}$ & 1428.90 & 83.48 \\
weighted degree $\langle s\rangle(\mathrm{min})$ & & & & \\
Average distance - & 177.87 & $\mathbf{1 8 8 . 9 2}$ & 113.16 & 18.24 \\
weighted degree $\langle\mathrm{s}\rangle(\mathrm{km})$ & 4 & 6 & $\mathbf{4}$ & 6 \\
Network diameter $(\mathrm{dG})$ & 2.088 & 2.53 & $\mathbf{2 . 2 6}$ & 3.11 \\
Average path length $(\langle l\rangle)$ & & & & \\
\hline
\end{tabular}

*. Cases in bold are closer to the aggregate layer's scores.

To obtain further insights into the topology of ACDN and its modal layers, we examine the geographical (spatial) distributions of the node measures of betweenness and closeness centrality, clustering coefficient, and degree, and the results are shown in the layouts of Figure 3 (layouts of Figure 3 are shown in more detail in the Appendix A). Betweenness centrality is a measure of intermediacy capturing the nodes' importance in terms of intermediating in network paths [32] while revealing the nodes undertaking the majority of network traffic when edge weights are considered as equal for all nodes $[28,30]$. As observed in Figure 3a (Figure A1, Appendix A), space seems to considerably affect the spatial distribution of betweenness centrality of ACDN. For instance, in the aggregate layer $\mathrm{ACDN}_{(\mathrm{A})}$, only one node (located centrally in the network) has a high betweenness centrality, while in the car $\mathrm{ACDN}_{(\mathrm{C})}$ and pedestrian $\mathrm{ACDN}_{(\mathrm{P})}$ layers, the color pattern 
appear more evenly distributed. The case of public transportation layer $\mathrm{ACDN}_{(\mathrm{T})}$ differs from the others, since nodes perform worse in the spatial distribution of this measure (more nodes belong to the red color scale). In general, the spatial distribution of betweenness centrality illustrates that central nodes in betweenness are also geographically central and located in the historic city center of Athens.
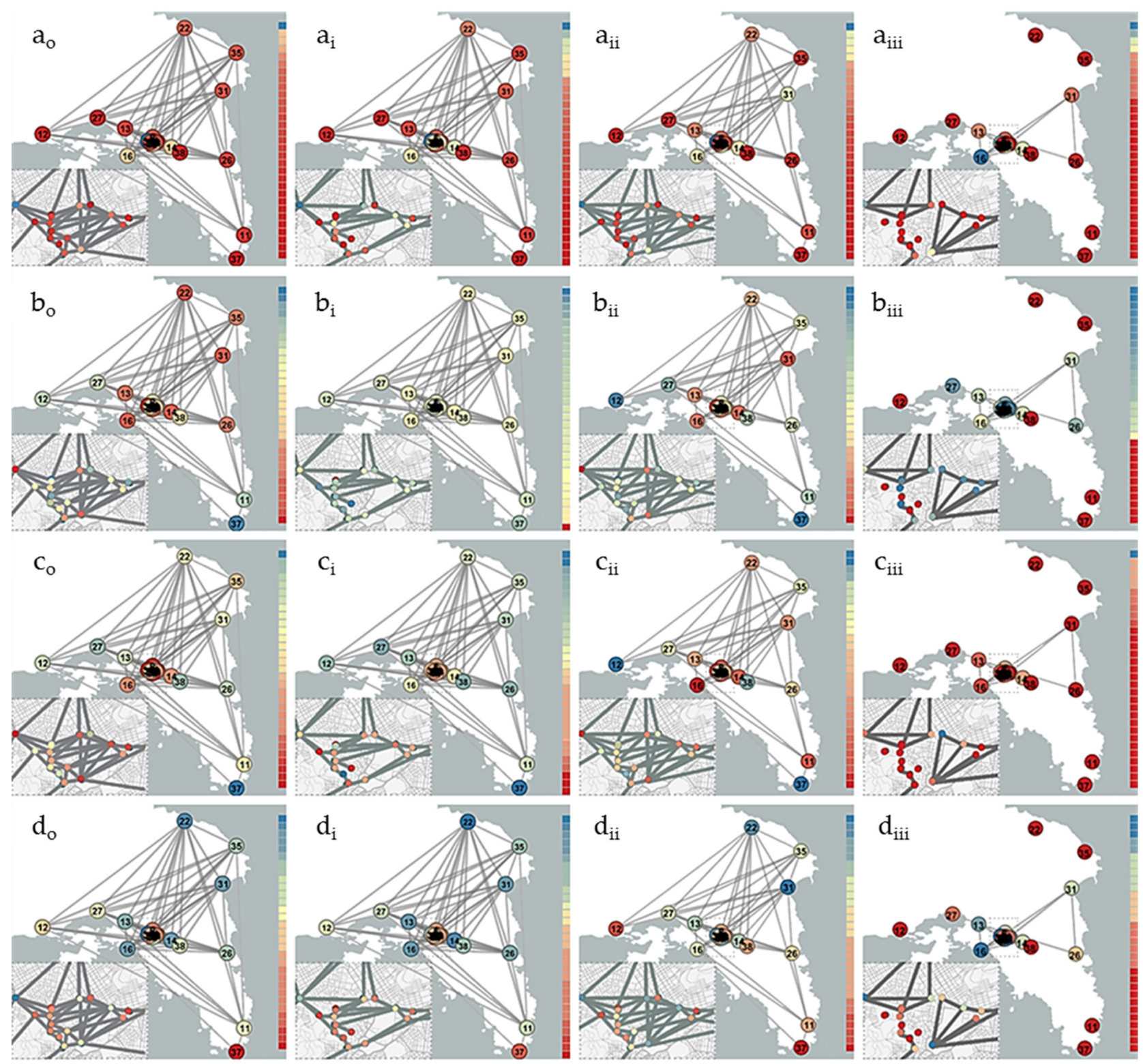

Figure 3. Spatial distributions of (a) betweenness centrality; (b) closeness centrality; (c) clustering coefficient; and (d) degree in the (o) aggregated layer; (i) car layer; (ii) pedestrian; and (iii) public transportation layer of Attica's cultural destinations network (ACDN). Max values are shown at the top and min at the bottom of each color ramp.

Next, closeness centrality expresses the reachability of the nodes in the network [32] and can be seen as a metric of the network's spatial impedance (cost) [30]. As observed in Figure $3 \mathrm{~b}$ (Figure A2, Appendix A), in the aggregate $\mathrm{ACDN}_{(\mathrm{A})}$ and pedestrian $\mathrm{ACDN}_{(\mathrm{P})}$ layers, geographically central nodes have a higher score in closeness than the peripheral ones that are accessible through longer paths and, therefore, higher transport costs. The same nodes seem to interact better in the car layer $\mathrm{ACDN}_{(\mathrm{C})}$ as they score higher, illustrating first the importance of private transport in the service of transportation $[5,8]$ and secondly the 
unreachable costs of accessing peripheral the destinations of ACDN on foot. Unfortunately, we can obtain a similar differential picture between the performance of the geographical core and the periphery in the closeness scores in the case of public transportation layer $\mathrm{ACDN}_{(\mathrm{T})}$. This picture highlights the lack of transportation support in the peripheral ACDN market, addressing avenues of public transportation planning towards servicing such valuable cultural destinations. The overall picture that we can obtain from the spatial distribution of closeness centrality is also favorable for the geographical core of the ACDN, where geographically central nodes are privileged in terms of connectivity since they are associated with low transport costs.

Next, the clustering coefficient is a measure expressing the "circularity" of information in networks [33] and, in the case of transportation networks, it has higher accessibility (more options to reach a node) in the network, both at the local and global levels [28,46]. In a recent work [46], the author provided evidence that a clustering coefficient can be representative for the level of regional urbanization and polycentric structure, which intuitively can be conceived on the basis that urbanized areas appear more star-like connected in the regional level. Within this context, we can observe in Figure 3c (Figure A3, Appendix A) that clustering is unevenly distributed across all layers, amongst which pedestrian layer $\mathrm{ACDN}_{(\mathrm{P})}$ resembles more to the aggregate $\mathrm{ACDN}_{(\mathrm{A})}$. In the car layer $\mathrm{ACDN}_{(\mathrm{C})}$, more nodes score high in clustering and more connected clusters appear, illustrating higher degrees of freedom offered by the private transport accessibility in contrast to the public transportation $\operatorname{ACDN}_{(\mathrm{T})}$, where almost no cluster is configured and nodes perform poorly (most belonging to the red scale) due to a lack of peripheral connectivity. In the context of its linkage with the level of urbanization [46], the spatial distribution of clustering coefficients provides insights into considering the pedestrian and public transportation modes in the service of the urbanized core of the ACDN market, whereas the mode of privet transport seems to operate more broadly in the service of the market of peripheral locations.

Finally, the spatial distribution of node degree illustrates the areas that are privileged in terms of network connectivity. As it can be observed in Figure 3d (Figure A4, Appendix A), the aggregate layer $\mathrm{ACDN}_{(\mathrm{A})}$ includes cases from all color palettes, illustrating a more balanced distribution. The cases of $\mathrm{ACDN}_{(\mathrm{C})}$ and $\mathrm{ACDN}_{(\mathrm{P})}$ illustrate a similar picture in terms of color range, although slight differences were expected due to fewer connections attached to these layers. Finally, in public transportation layer $\operatorname{ACDN}_{(\mathrm{T})}$, the overall picture of the spatial distribution of degree shapes a more distinguishable geographically centered hub-and-spoke $[8,28,30]$ pattern, where degree hubs are located in the city center and coincide with transport hubs, while the rest of the active nodes are connected directly to these hubs. To shape a deeper picture of connectivity and obtain insights into pattern recognition of the ACDN, we examine the degree distributions of the aggregate and its modal layers, and the results of this analysis are shown in the scatter plots of Figure 4 , with the best possible fitting curves in terms of determination.

As it can be observed, the degree distributions of aggregate $\operatorname{ACDN}_{(A)}$ and pedestrian $\mathrm{ACDN}_{(\mathrm{P})}$ layers shape (in the best possible determination) one-term Gaussian (bell-shaped) patterns, implying that the majority of nodes have a degree close to the layer's average. Although of marginal determination, this is a typical pattern describing spatial networks of lattice-like topology $[30,37]$, which are intensively ruled by spatial constraints and planarity [28], something expected for the pedestrian case but not absolutely for the overall ACDN market. This observation verifies the findings in the previous parts of the analysis about the structural and topological similarity between $\operatorname{ACDN}_{(A)}$ and $\operatorname{ACDN}_{(\mathrm{P})}$ layers. On the other hand, the degree distribution of car layer $\mathrm{ACDN}_{(\mathrm{C})}$ best fits a trigonometric (cosine) curve. Within the context of network science $[27,41]$, such patterns do not correspond to a typical network topology. However, within the range of the empirical $A C D N_{(C)}$ degree distribution interval, this cosine curve decomposes into a pair of bell-shaped patterns, thus illustrating the emergence of two Gaussian behaviors at different degree scales: The first is centered around an average degree of $4^{+}$and the second around $11^{-}$. This observation reveals a tendency of $\mathrm{ACDN}_{(\mathrm{C})}$ to divide into two communities (to be verified at the next 
part of analysis): one highly connected probably referring to the urban core, and a second one less connected probably referring to the periphery. Overall, this finding may illustrate a good performance of the private transportation mode to adjust in geographical space, providing avenues of further research towards this research hypothesis.
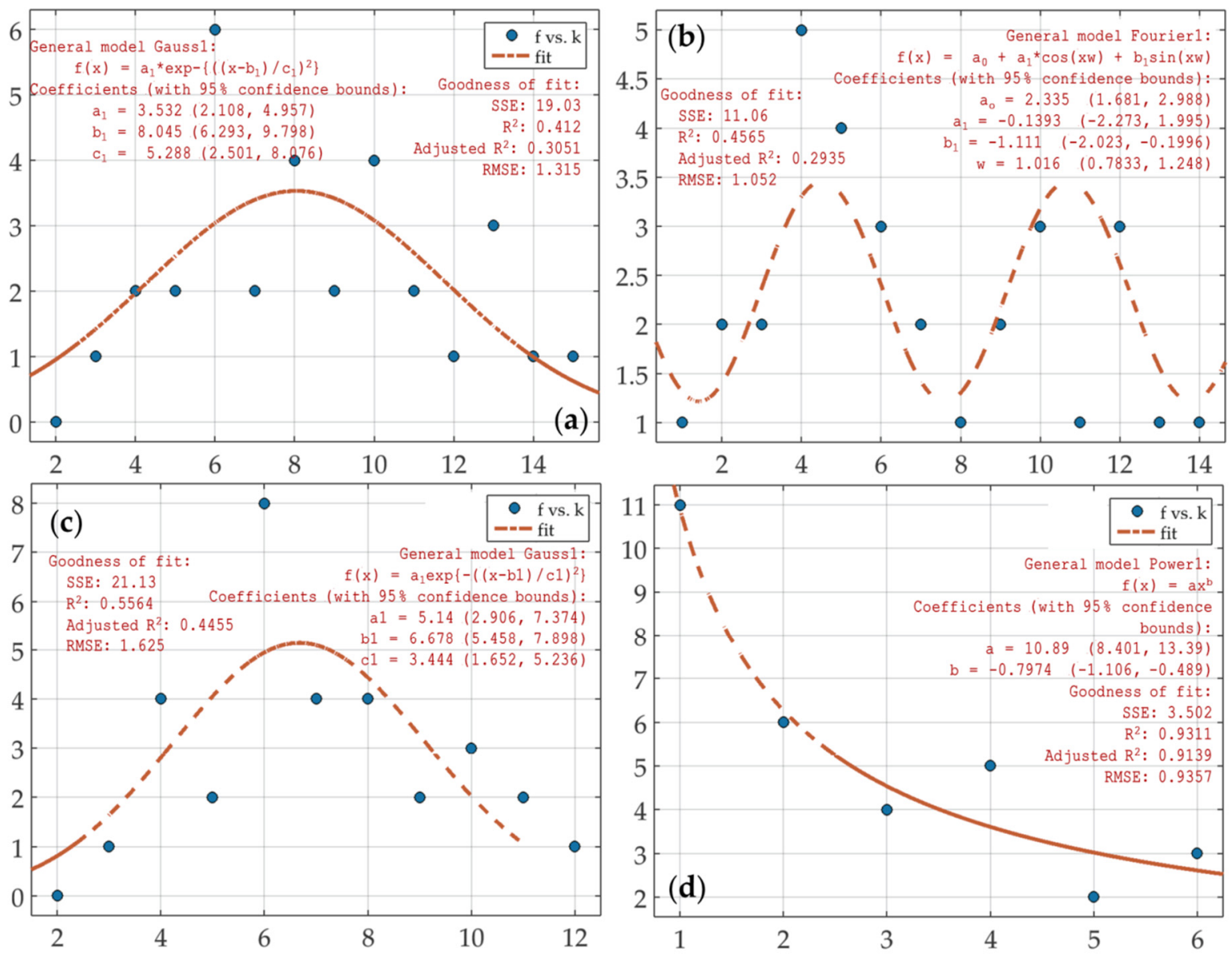

Figure 4. Degree distributions of the (a) aggregate $\left(\mathrm{ACDN}_{(\mathrm{A})}\right)$; (b) car $\left(\mathrm{ACDN}_{(\mathrm{C})}\right)$; (c) pedestrian $\left(\mathrm{ACDN}_{(\mathrm{P})}\right)$; and $(\mathrm{d})$ public transportation $\left(\mathrm{ACDN}_{(\mathrm{T})}\right)$ layers of Attica's cultural destinations network $(\mathrm{ACDN})$.

Finally, the degree distribution of public transportation layer $\mathrm{ACDN}_{(\mathrm{T})}$ follows (in a high determination) a power-law pattern, illustrating first a scale-free property and verifying next the hub-and-spoke topology previously observed for this layer (in the spatial distributions examination). Generally, hub-and-spoke topology is an optimized outcome driven by the preferential attachment growth mechanism $[41,42]$ and, within the context of optimization, it describes a good structure of hierarchy in the allocation of the public transportation resources serving the ACDN market. However, the claim of optimization cannot also stand for the case of this layer's functionality, since this layer is not fully connected and, therefore, not all nodes are accessible through a path served by public transport. Overall, this finding reveals a "least-cost" strategy ruling the tourism development policy, addressing avenues of further research towards the direction of improving cultural tourism network accessibility while minimizing its transportation cost. In a further pattern recognition approach, we examine the sparsity (spy) plots of ACDN, shown in Figure 5. As it can be observed, the sparsity patterns of the aggregate $A C D N_{(A)}$ and pedestrian $A C D N_{(P)}$ layers are more similar, verifying previous topological and degree distribution findings. Moreover, the spy plot of car layer $\operatorname{ACDN}_{(C)}$ illustrates two distinct connectivity regions (separated by an axis parallel to the secondary diagonal), verifying previous observations 
of degree distribution analysis. Finally, the spy plot of the public transport layer $\operatorname{ACDN}_{(C)}$ illustrates its insufficient connectivity, since connections in this layer are clustered at the top-left quadrant of the adjacency.

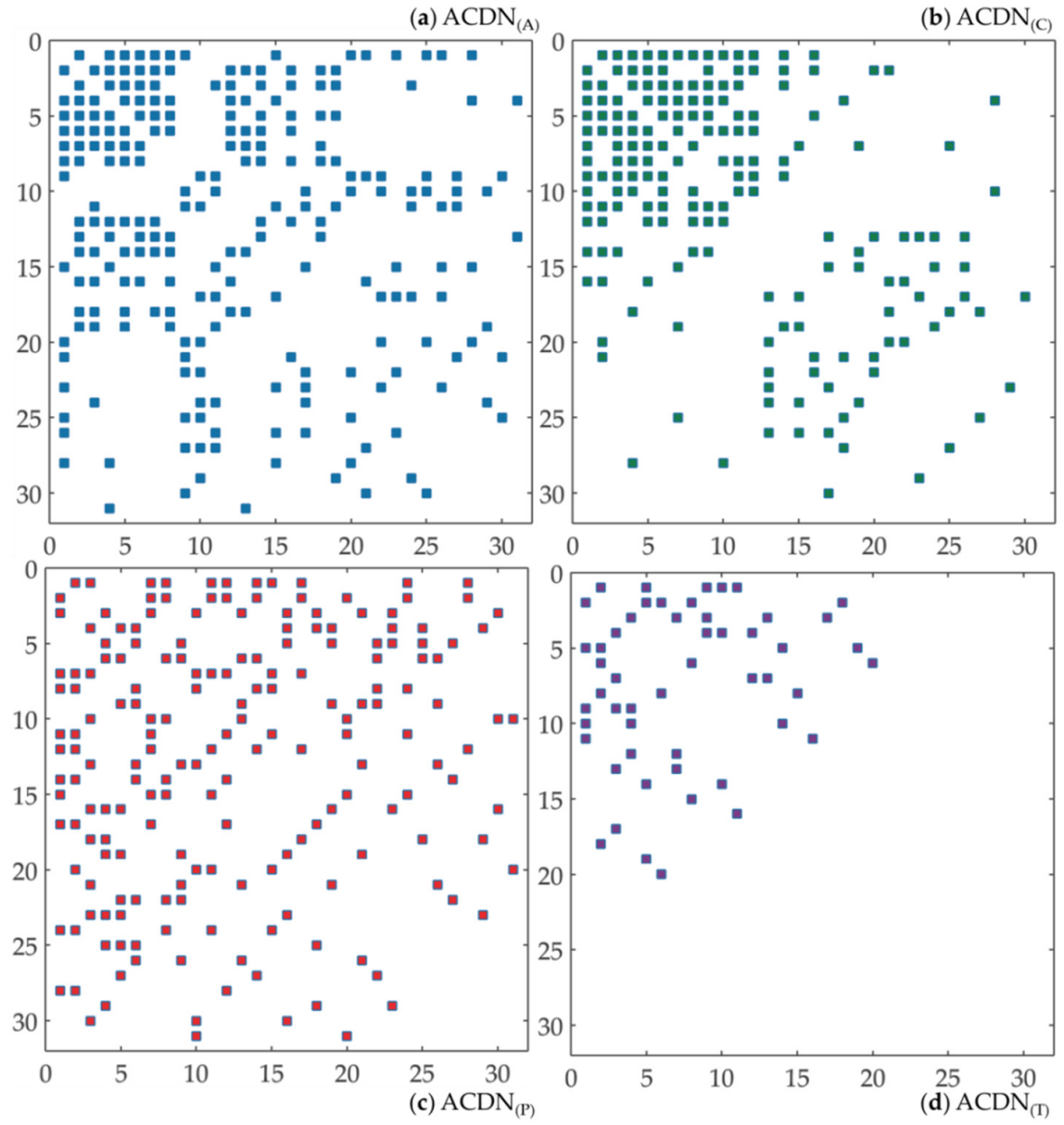

Figure 5. Sparsity (spy) plot of the adjacencies of the aggregate ACDN and its layers (node ordering according to node degree).

In terms of network topology, (i) a concentration in a corner or a cross-oriented concentration in the adjacency matrix may provide insights into a hub-and-spoke topology, (ii) a linear concentration along the main diagonal may provide insights into a latticelike topology, and (iii) a scattered sparsity distribution may provide insights into either a random-like or lattice-like topology depending on the node ordering [30,37]. Within this context, the cases shown in Figure 4 comply with previous pattern distribution findings (Figure 3), providing evidence for considering a hub-and-spoke pattern for the topology of public transport layer $\mathrm{ACDN}_{(\mathrm{T})}$ and lattice-like patterns elsewhere.

\subsection{Community Detection}

This part applies community detection analysis to the aggregate layer $\operatorname{ACDN}_{(\mathrm{A})}$ based on the modularity optimization algorithm [34] in order to quantitatively examine the network potential to subdivide into communities. The analysis applies to the aggregate layer because it is a multimodal (and, thus, more integrated) layer, including all available transport modes, and connected layer; therefore, it is unbiased in terms of baffling network components with modularity communities. The results of the analysis are shown in Figure 6, where it can be observed that the topology of ACDN bisects into two communities: The first including the urban core, consisting of 17 cultural destinations (red nodes), and the second one including peripheral locations, consisting of 14 cultural destinations (blue nodes). This result appears 
first to be geographically driven, since it is more strongly determined by spatial constraints, resulting in the emergence of a Central $\left(C_{1}\right)$ and Peripheral $\left(C_{2}\right)$ community.

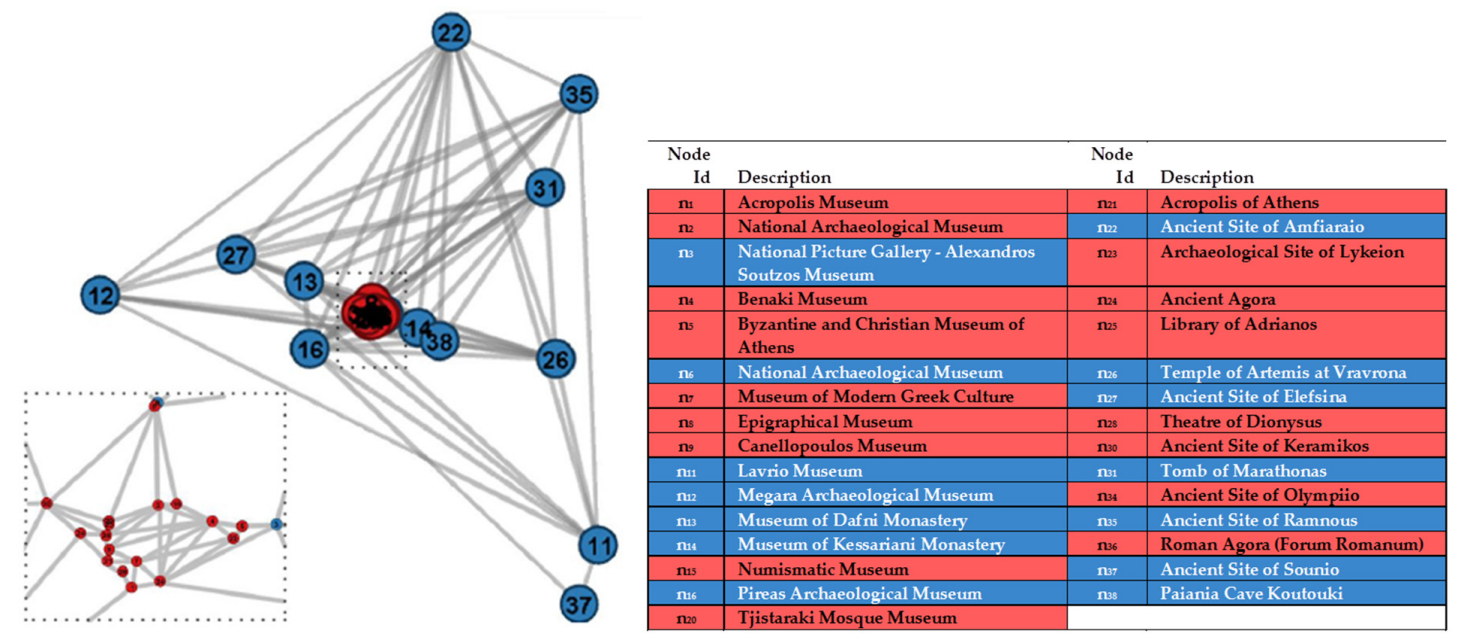

Figure 6. Communities extracted from the community detection analysis of ACDN under the modularity optimization algorithm.

To further examine whether community detection ACDN is also driven by modal configuration forces (car, pedestrian, and public transport functionality), we computed network measures for the aggregate $\mathrm{ACDN}_{(\mathrm{A})}$ and modal layers $\mathrm{ACDN}_{(\mathrm{C})}, \mathrm{ACDN}_{(\mathrm{P})}$, and $\operatorname{ACDN}_{(\mathrm{T})}$, and the results are shown in Table 4. As observed, in the case of the urban core community $\left(C_{1}\right)$, the total amount of network measures of the pedestrian layer $A_{C D N}(P)$ is numerically closer (in absolute terms) to the scores of aggregate layer $\operatorname{ACDN}_{(\mathrm{A})}$, whereas, in the case of the peripheral community, all measures of car layer $\operatorname{ACDN}_{(\mathrm{A})}$ are closer (in absolute terms) to the aggregate scores. This result reveals that the communities emerged by the modularity optimization algorithm are also modally configured, where the pedestrian mode appears determinative for the configuration of the urban core community and the car mode of the peripheral community. The absence of relevance between the aggregate $\mathrm{ACDN}_{(\mathrm{A})}$ and the public transportation $\mathrm{ACDN}_{(\mathrm{T})}$ layers can be seen as an aspect of deficiency in the service of the ACDN market by public transportation, addressing avenues of further research into the improvement of participation of public transport in the service of ACDN accessibility.

\subsection{Empirical Analysis}

At the final step of analysis, we aim to detect distinguishable attributes in modularity communities generated at the previous step. This approach builds on statistical testing using error bars of $95 \%$ confidence intervals (CIs) for the means of (i) a set of topological node attributes (extracted from Table 1) and (ii) the tourism-defined socioeconomic attributes of Table 2. The results of the analysis are shown in Figure 7 for topological attributes and in Figure 8 for tourism-defined socioeconomic attributes. As observed in Figure 7, the only statistical difference between the urban core $\left(C_{1}\right)$ and the peripheral community $\left(C_{2}\right)$ of $\mathrm{ACDN}$ is the average time-weighted degree, where the central community performs with lower scores as expected due to the geographical concentration in the city center. However, this is not the case for the average distance-weighted degree, where, first, no significant difference was detected between the communities and, secondly, nodes in the peripheral community tend to have less average distance-weighted degree than the urban core. This observation illustrates a balance between the jointed effect of geography and connectivity in both communities, implying that both communities undertake similar loadings of spatial impedance compared to their connectivity. This result can be more comprehensive by recalling the results of Table 4, according to which the average degree of the peripheral community is $50 \%$ greater than the urban core community so that this higher connectivity 
potential of $\mathrm{C}_{2}$ can reflect the distance-weighted degree. As far as the other topological measures are concerned, no statistical differences were detected. However, the slight differences emerging between cases illustrate a tendency of $(i)$ a more centralized pattern ruling the topology of the central community, (ii) a more distant configuration of the peripheral community, and (iii) a better intermediacy of the central community that is in line with the empirical knowledge of planar spatial networks [28].

Table 4. Network measures computed for the central and peripheral ACDN communities generated by the modularity optimization algorithm.

\begin{tabular}{ccccc}
\hline \multicolumn{5}{c}{ A. Central (Urban Core) Community of the ACDN } \\
\hline & $\mathrm{ACDN}_{(\mathrm{A})}$ & $\mathrm{ACDN}_{(\mathrm{C})}$ & $\mathrm{ACDN}_{(\mathrm{P})}$ & $\mathrm{ACDN}_{(\mathrm{T})}$ \\
\hline Edges & 46 & 24 & $\mathbf{4 4}$ & 12 \\
Graph Density & 0.338 & 0.175 & $\mathbf{0 . 3 2 4}$ & 0.088 \\
Average Degree & 5.412 & 2.824 & $\mathbf{5 . 1 7 6}$ & 1.412 \\
Average Weighted Time & 41.059 & 12.941 & $\mathbf{6 2 . 5 8 8}$ & 17.059 \\
Average Weighted Distance & 4.709 & 3.688 & $\mathbf{4 . 7 8}$ & 1.959 \\
Network Diameter & 3 & 6 & $\mathbf{3}$ & $\mathbf{3}$ \\
Average Path Length & 1.801 & 2.592 & $\mathbf{1 . 8 6}$ & 1.692 \\
Clustering Coefficient & 0.565 & 0.438 & $\mathbf{0 . 5 8 9}$ & 0.444 \\
\hline & B. Peripheral Community of the ACDN & & \\
\hline Edges & $\mathrm{ACDN}$ & $\mathrm{ACDN}$ & $\mathrm{ACDN}$ & \\
\hline Graph Density & 57 & 55 & 40 & $\mathrm{ACDN}$ \\
Average Degree & 0.626 & $\mathbf{0 . 6 0 4}$ & 0.44 & 8 \\
Average Weighted Time & 8.143 & $\mathbf{7 . 8 5 7}$ & 5.714 & 0.088 \\
Average Weighted Distance & 490 & $\mathbf{3 4 4 . 8 5 7}$ & 2934.286 & 133.286 \\
Network Diameter & 354.443 & $\mathbf{3 7 8 . 6 5 7}$ & 232.486 & 31.657 \\
Average Path Length & 3 & $\mathbf{3}$ & $\mathbf{3}$ & 4 \\
Clustering Coefficient & 1.395 & $\mathbf{1 . 4 1 8}$ & 1.637 & 1.905 \\
\hline
\end{tabular}

*. Cases in bold are closer to the aggregate layer's scores.
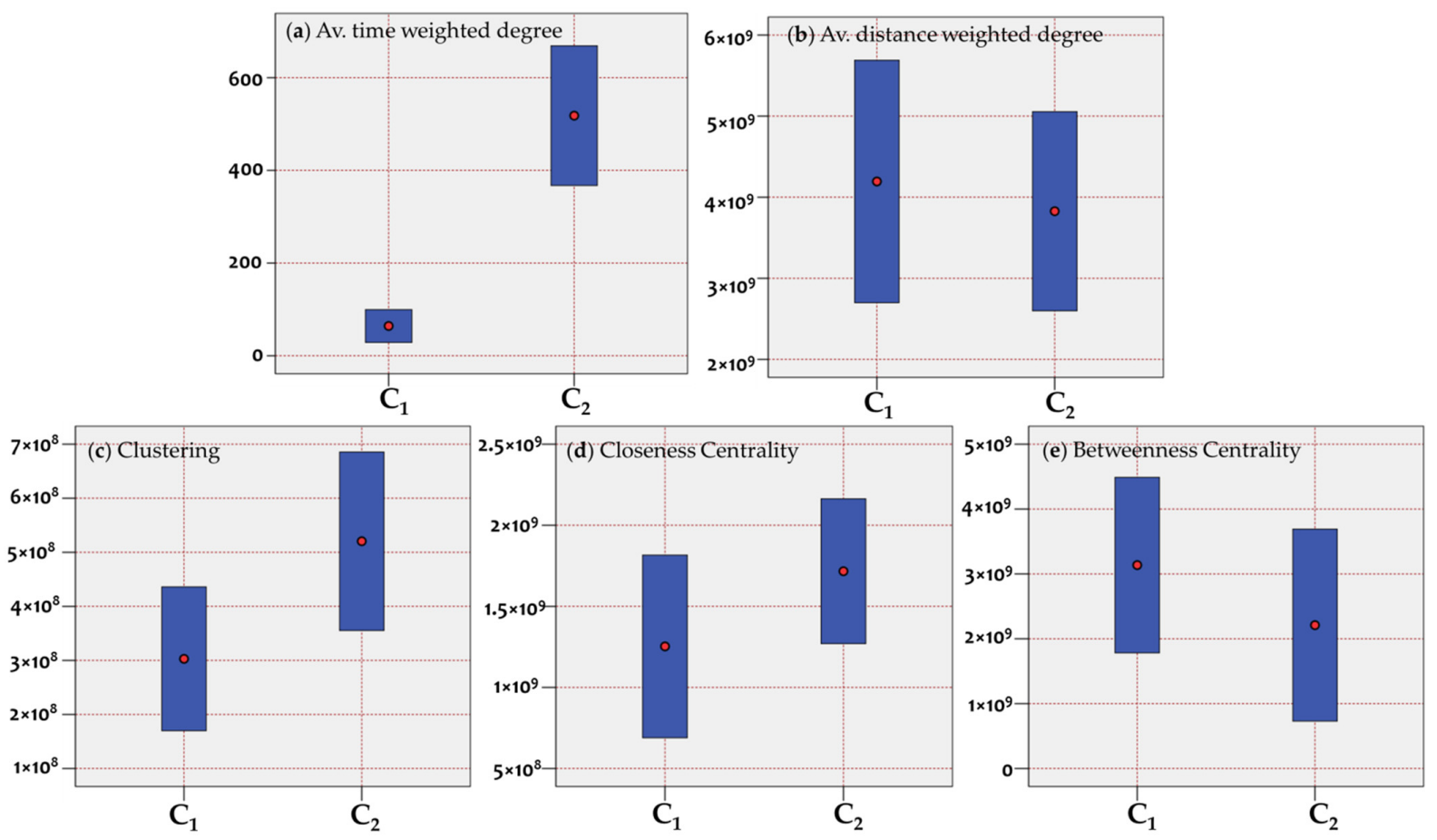

Figure 7. Error bars of $95 \%$ CIs for the mean values of ACDN topological node attributes of (a) average weighted degree (time), (b) average weighted degree (distance), (c) clustering coefficient, (d) closeness centrality, and (e) betweenness centrality computed per modularity community. 


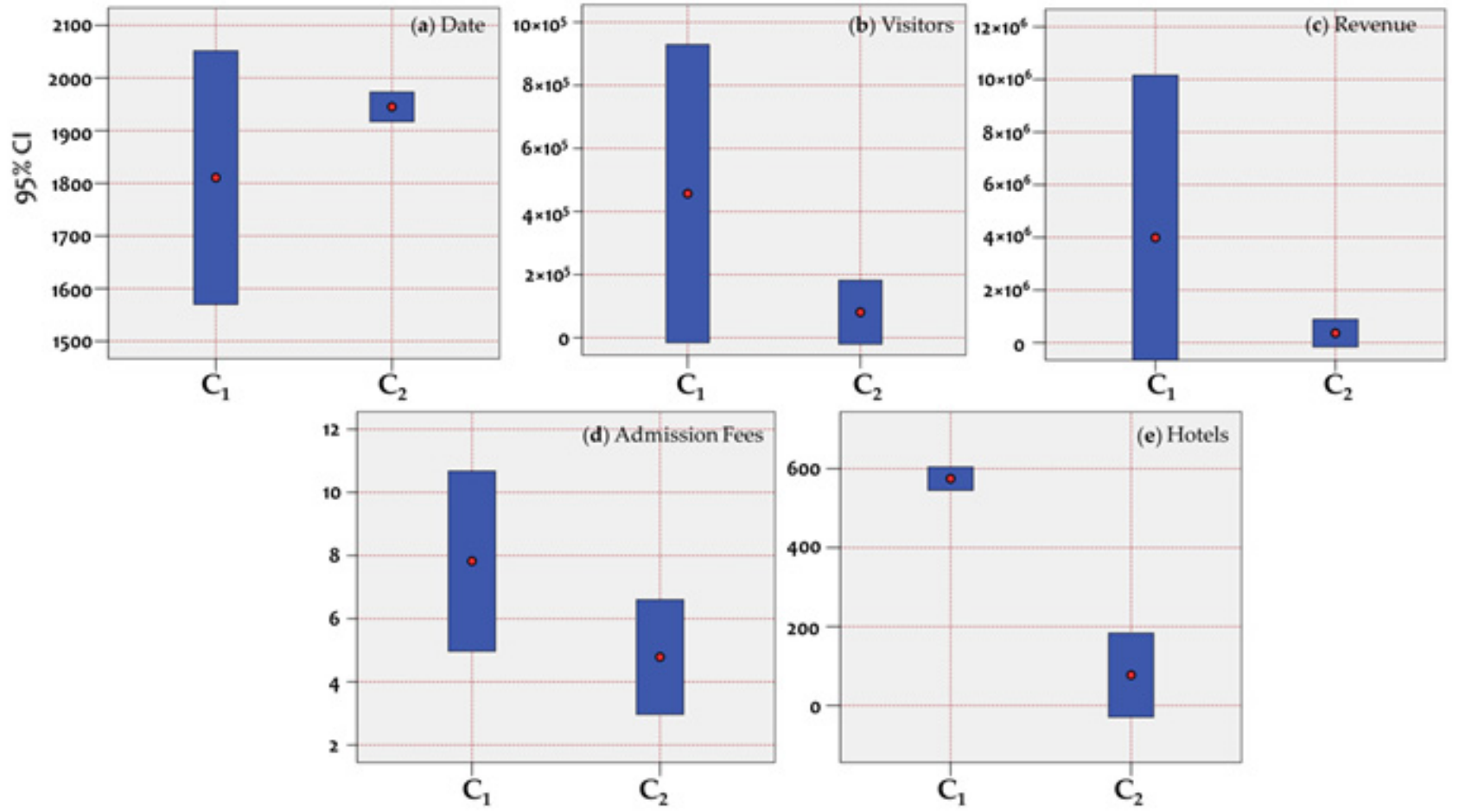

Figure 8. Error bars of $95 \%$ CIs for the mean values of the ACDN tourism node attributes (a) cultural tourism destination (CTD) establishment date, (b) CTD's number of visitors, (c) CTD's annual revenue, (d) CTD's admission fees, and (e) CTD's betweenness centrality computed per modularity community.

On the other hand, according to Figure 8, the only statistical difference between the ACDN communities stands for the number of neighbor hotels, illustrating the dominance in the attractiveness of the urban core compared to the periphery of the ACDN. This result, in conjunction with the hub-and-spoke topology of $\mathrm{ACDN}_{(\mathrm{T})}$ that was previously detected, illustrates also a profit-oriented strategic plan of public transportation policy in the service of the cultural destinations market in Attica. In terms of the information that can be retrieved by reading tendencies in the insignificant differences of Figure 8 , we can assume that the peripheral nodes (destinations) tend to be newer entries in the ACDN market, and the tendency of the central community to attract more visitors is accompanied with a tendency to create higher revenue and to require higher admission fees. In terms of variance, we can observe higher homogeneity (to the extent that is expressed by the CI lengths) describing all tourism-oriented socioeconomic attributes of the peripheral community, except the number of hotels, where the urban core community is more homogenous (illustrating another aspect of the urban core attractiveness).

\section{Discussion}

Tourism is a multidimensional global phenomenon, as it is associated with concepts such as culture, development, and economy; thus, spatial planning is a complex and interdisciplinary process [47]. In an ever-changing environment, formal tourism agencies and communities are interested in spatial tourism planning and expect innovative proposals from scientific research $[4,48]$. As presented so far, tourism is one of the main axes of development in Greece [4,49], where a strong advantage of the Greek tourism product is the available cultural capital, which attracts both investments in the tourism sector and tourists $[3,47,50]$. After all, cultural motivation is one of the strongest factors that drive people to travel [47], and even when it is not the sole cause of travel, it often acts as a complement to other motivations. In the complex nature of tourism, space is a major dimension affecting local economies and communities [8,51], where the demand of 
studying the location and development of infrastructures to support tourism in an area (transport infrastructure, accommodation, etc.) is a multidisciplinary requirement involving the diverse conceptualization of spatial planning, tourism geography and economics, regional science, and similar disciplines, both at the scientific and policy levels [51,52]. As a discipline serving complexity, network science equips tourism research with tools to study and understand complex systems of demand and supply of tourism services in the context of the network paradigm [29]. In an attempt to combine tourism geography and network science, this paper focused on the case of cultural tourism destinations of Attica, Greece, and evaluated the capacity of different transportation modes to serve accessibility demand in this cultural tourism market. Thus, car, pedestrian, and public transportation were considered as different layers in a multilayer network model, analyzing their role in a complex network setting. The analysis detected differences and similarities in the structure and functionality of these layers and, in particular, revealed the following: (i) the same nodes in the same geographical context interact differently with each other at each of the levels; (ii) car and pedestrian layers share common topological and geometric (geographical) features, a fact that can be attributed to the parallel space shared by the pedestrian and road accessibility; however, differences in the accessibility speeds resulted in different spatial connection weights and, thus, different weighted topological features; (iii) the topology of the public transport level appeared completely different than of the other layers and was described by a hub-and-spoke pattern, describing a good structure of hierarchy in the allocation of public transportation resources and a profit-oriented strategic plan of public transportation policy in the service of the cultural destinations market in Attica; (iv) the cultural destination network in Attica can decompose into two modularity communities, an urban core consisting of the geographically central nodes and a peripheral community consisting of regional nodes. Their behavior differs both in topological measures and in the tourism-oriented attributes describing node properties in the physical network. By conducting the analysis, the central community emerged as more cohesive, cost-effective, and had more tourism infrastructure, while the peripheral community was more accessible. However, the shorter operating time of the junctions and the absence of hotel infrastructure did not enhance the tourist attractiveness of the regional junctions of the cultural network of Attica; (v) community detection was not only driven by inevitable geographical constraints but also by the network layer's functionality, where the pedestrian layer prevailed in the configuration of the urban core community and the car layer of the peripheral community; and (vi) the public transport layer did not appear to have a dominant role in the configuration of the cultural destinations network topology, acting supplementarily to the other two modes. Its role illustrated an aspect of deficiency in the service of the accessibility of such valuable cultural tourism markets.

Overall, community detection and different community profiles that emerged, in the context of the cultural tourism market, underlined different needs in the planning and management of cultural tourism in Attica. In the central community, where the pedestrian is the predominant mode, complemented by public transport, it appears more appropriate to improve connectivity in the physical network by route management for a better, cost-effective navigation, through its nodes. At the same time, the design of the public transportation's cultural itineraries and their modernization could enhance mobility within the urban core community. As participation in walking routes and the use of tourist buses for sightseeing is already widespread among tourists in Athens, such practices are expected to be efficient in upgrades and with respect to sustainability of this central tourism market; thus, it is recommended for official authorities. On the other hand, the different profiles of the peripheral community suggest another direction of policy practices towards sustainable tourism development. The car seems to be the right means to move this community, which, in combination with the reduced supply of tourist beds, implies an inability of the destinations (nodes) of this community to meet average tourist needs. In addition, despite the detected tendency of community nodes to cluster, the design and construction of roundabouts linking them can become effective in the context that they 
will accompany a decrease in access time. Another direction of improvement is a better promotion strategy of the nodes (museums and archeological sites) of this community that is expected to results in greater integration of these nodes in the cultural network of Attica. Strengthening the connections with the central node community to the extent that the central community will act as a hub for the regional nodes and organizing one-day excursion packages to the peripheral nodes are also expected to increase the currently low traffic of the nodes.

\section{Conclusions}

The different profiles of the communities in the cultural tourism market, as described in this paper, underline the different needs that exist in terms of planning and managing tourist mobility in the network of cultural destinations in Attica (ACDN) concerning the available infrastructure. The evaluation of the already existing infrastructures, the correlation with the nodes' geographical location and dynamics, and the supply of intermodal mobility will result in an effective transport system that serves the demands of the cultural network's visitors. The challenges for sustainable mobility indicate a different approach to the development of cultural tourism that does not take into account only the area's cultural capital. In conclusion, the cultural destination network of Attica, Greece, can be modeled as a graph in the context of the accessibility of places. However, the individual layers of the network compete to serve the demand for mobility between the nodes due to their different topologies and functionalities. According to the network analysis, the network of cultural destinations in Attica can be divided into two communities, a central and a peripheral one, with different topological, geographical, and functional features. The central community is better served by public transport, which makes it easier to visit its nodes. On the contrary, the peripheral community is poorly supported by the transport system and visiting it requires effort and private transport modes. Finally, despite the enhanced identity that Attica has in the cultural tourism market, transportation infrastructure is not sufficient for the visit of the nodes, and they can be further developed. In conclusion, the transition from theoretical and scientific approaches to policy making is a major stake in spatial planning. The results of this analysis can be used to formulate policies that will organize and manage the tourism mobility of a destination's network in relation to the available infrastructure. The evaluation of the existing infrastructure, their correlation with the geographical location and dynamics of the nodes, and the interconnection of the means of transport will result in a better utilization of the possibilities of the transport system in relation to the cultural network. After all, cultural capital alone is not enough to develop tourism, as modern challenges place emphasis on sustainable mobility.

Author Contributions: Conceptualization, A.K. and D.T.; methodology, D.T. and S.P.; software, A.K. and D.T.; validation, D.T. and S.P.; formal analysis, A.K.; investigation, A.K.; resources, A.K. and D.T.; data curation, A.K. and D.T.; writing-original draft preparation, A.K. and D.T.; writing-review and editing, D.T.; visualization, A.K. and D.T.; supervision, D.T. and S.P.; project administration, D.T. All authors have read and agreed to the published version of the manuscript.

Funding: This research received no external funding.

Institutional Review Board Statement: Not applicable.

Informed Consent Statement: Not applicable.

Data Availability Statement: Hellenic Statistical Authority-ELSTAT. (https: / / www.statistics.gr/ en/statistics/-/publication/SCI21/2019-M12 (accessed on 25 May 2020)).

Acknowledgments: The authors thank the two anonymous reviewers for their constructive comments and positive disposition.

Conflicts of Interest: The authors declare no conflict of interest. 


\section{Appendix A}

Table A1. The 31 cultural destinations composing the node set of the multiplex ACDN.

\begin{tabular}{|c|c|c|}
\hline Node no. & $\begin{array}{c}\text { Label } \\
\text { (Node ID) }\end{array}$ & Description \\
\hline 1 & $\mathrm{n}_{1}$ & Acropolis Museum \\
\hline 2 & $\mathrm{n}_{2}$ & National Archaeological Museum \\
\hline 3 & $\mathrm{n}_{3}$ & National Picture Gallery-Alexandros Soutzos Museum \\
\hline 4 & $\mathrm{n}_{4}$ & Benaki Museum \\
\hline 5 & $\mathrm{n}_{5}$ & Byzantine and Christian Museum of Athens \\
\hline 6 & $\mathrm{n}_{6}$ & National Archaeological Museum \\
\hline 7 & $\mathrm{n}_{7}$ & Museum of Modern Greek Culture \\
\hline 8 & $\mathrm{n}_{8}$ & Epigraphical Museum \\
\hline 9 & $\mathrm{n}_{9}$ & Canellopoulos Museum \\
\hline 10 & $\mathrm{n}_{11}$ & Lavrio Museum \\
\hline 11 & $\mathrm{n}_{12}$ & Megara Archaeological Museum \\
\hline 12 & $\mathrm{n}_{13}$ & Museum of Dafni Monastery \\
\hline 13 & $\mathrm{n}_{14}$ & Museum of Kessariani Monastery \\
\hline 14 & $\mathrm{n}_{15}$ & Numismatic Museum \\
\hline 15 & $\mathrm{n}_{16}$ & Pireas Archaeological Museum \\
\hline 16 & $\mathrm{n}_{20}$ & Tjistaraki Mosque Museum \\
\hline 17 & $\mathrm{n}_{21}$ & Acropolis of Athens \\
\hline 18 & $\mathrm{n}_{22}$ & Ancient Site of Amfiaraio \\
\hline 19 & $\mathrm{n}_{23}$ & Archaeological Site of Lykeion \\
\hline 20 & $\mathrm{n}_{24}$ & Ancient Agora \\
\hline 21 & $\mathrm{n}_{25}$ & Library of Adrianos \\
\hline 22 & $\mathrm{n}_{26}$ & Temple of Artemis at Vravrona \\
\hline 23 & $\mathrm{n}_{27}$ & Ancient Site of Elefsina \\
\hline 24 & $\mathrm{n}_{28}$ & Theatre of Dionysus \\
\hline 25 & $\mathrm{n}_{30}$ & Ancient Site of Keramikos \\
\hline 26 & $\mathrm{n}_{31}$ & Tomb of Marathonas \\
\hline 27 & $\mathrm{n}_{34}$ & Ancient Site of Olympiio \\
\hline 28 & $\mathrm{n}_{35}$ & Ancient Site of Ramnous \\
\hline 29 & $\mathrm{n}_{36}$ & Roman Agora (Forum Romanum) \\
\hline 30 & $\mathrm{n}_{37}$ & Ancient Site of Sounio \\
\hline 31 & $\mathrm{n}_{38}$ & Paiania Cave Koutouki \\
\hline
\end{tabular}

Source: On elaboration based on data of the Greek Ministry of Culture.
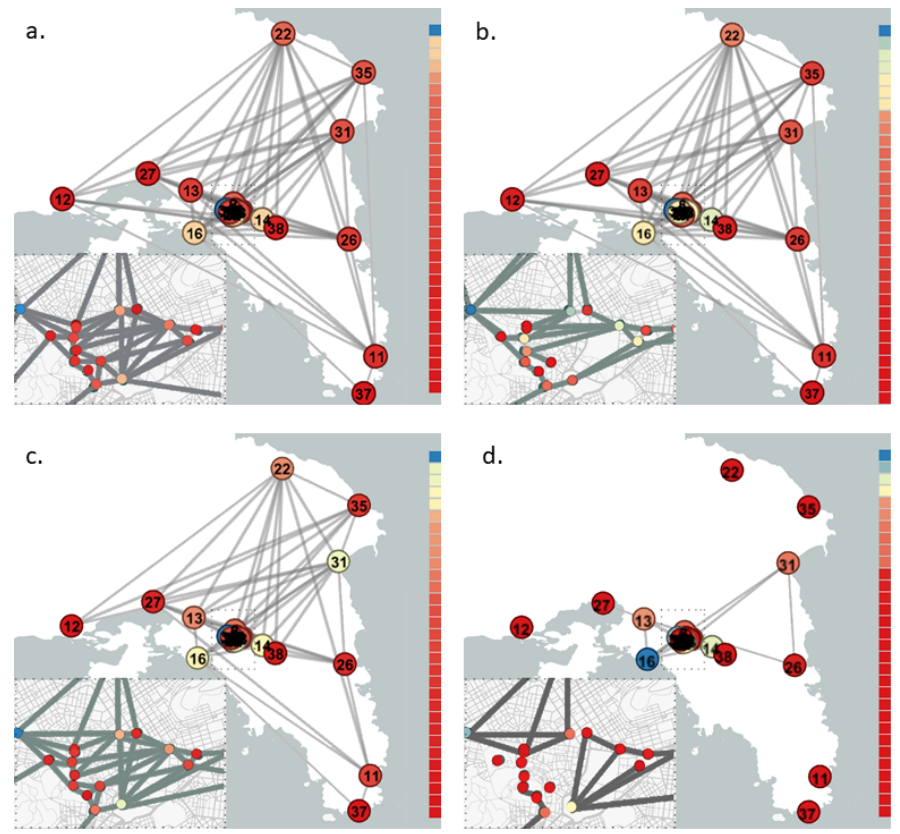

Figure A1. Spatial distribution of betweenness centrality in the (a) aggregate layer; (b) car layer; (c) pedestrian; and (d) transportation layer (max values are shown on the top and min at the bottom of each color ramp). 

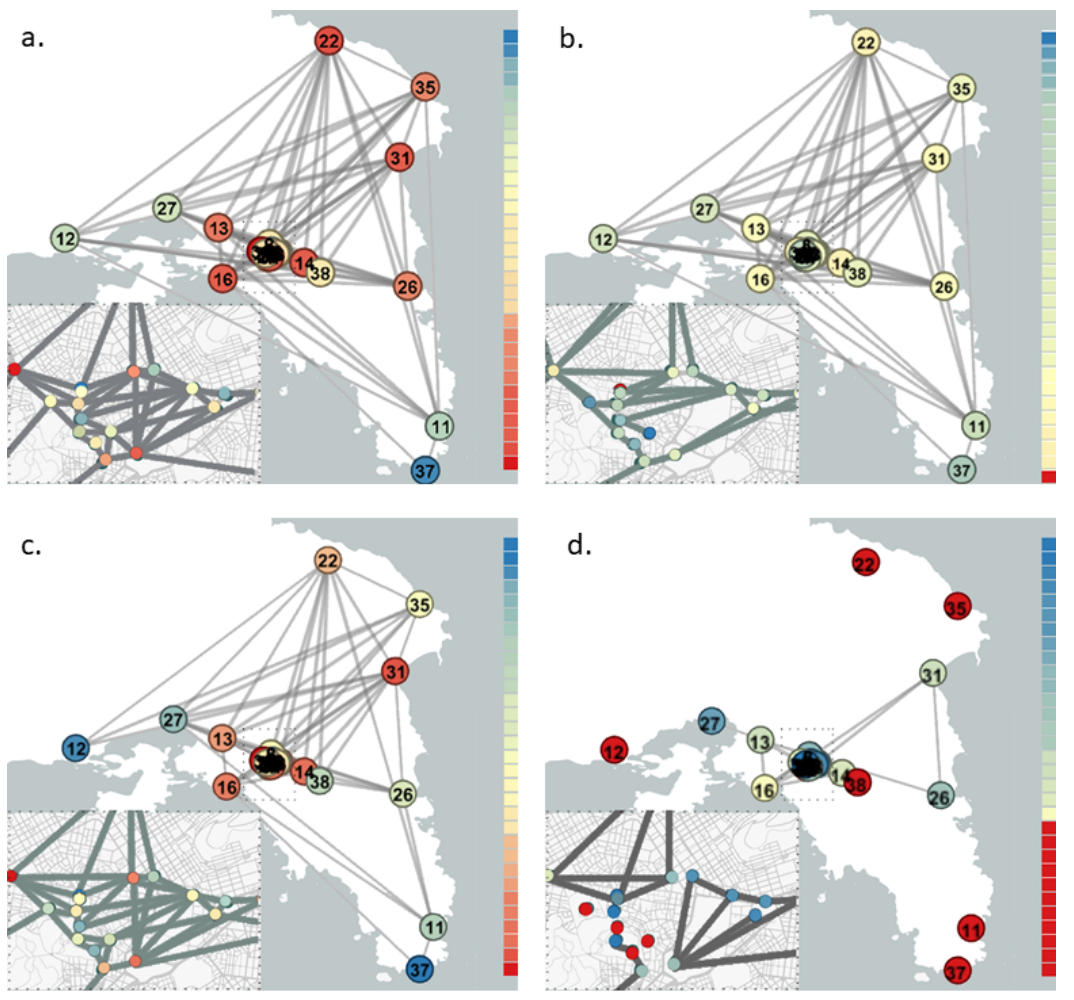

Figure A2. Spatial distribution of closeness centrality in the (a) aggregate layer; (b) car layer; (c) pedestrian; and (d) transportation layer (max values are shown on the top and min at the bottom of each color ramp).
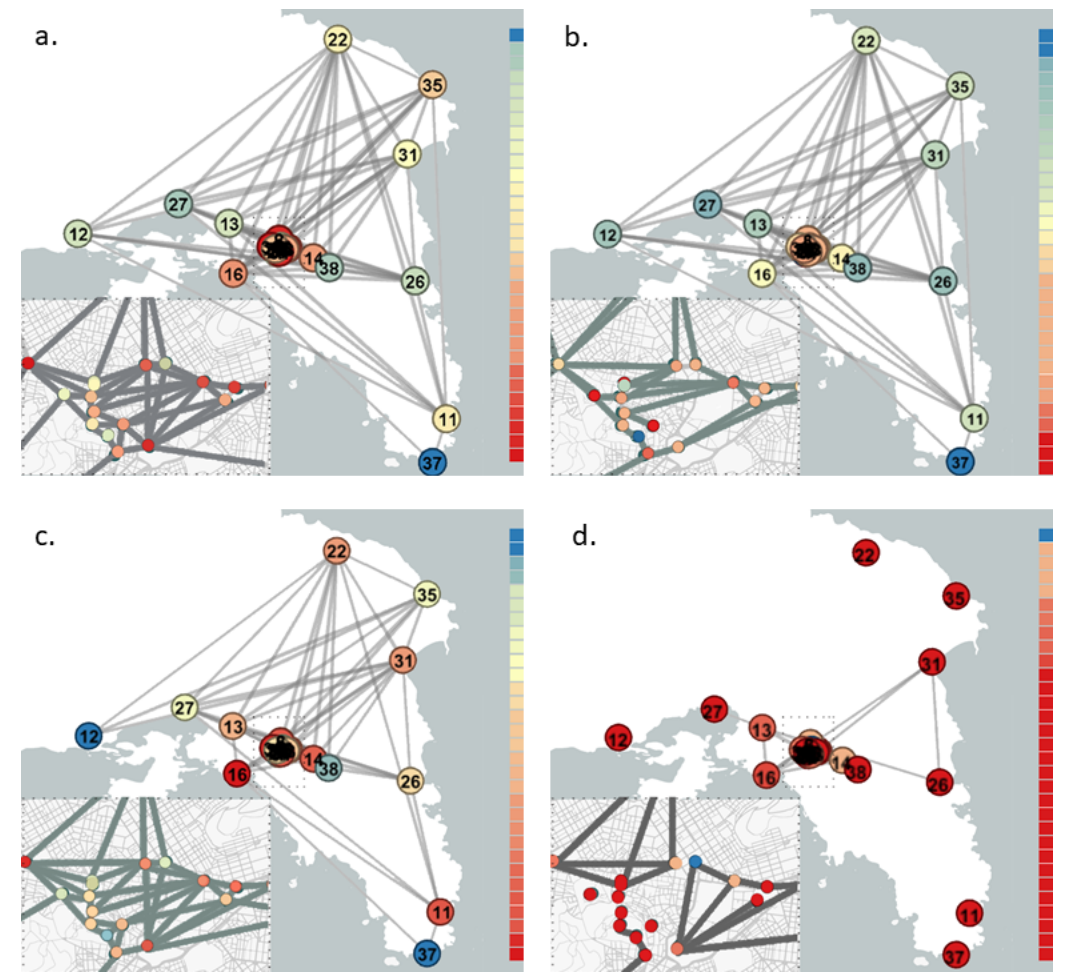

Figure A3. Spatial distribution of the clustering coefficient in the (a) aggregate layer; (b) car layer; (c) pedestrian; and (d) transportation layer (max values are shown on the top and min at the bottom of each color ramp). 

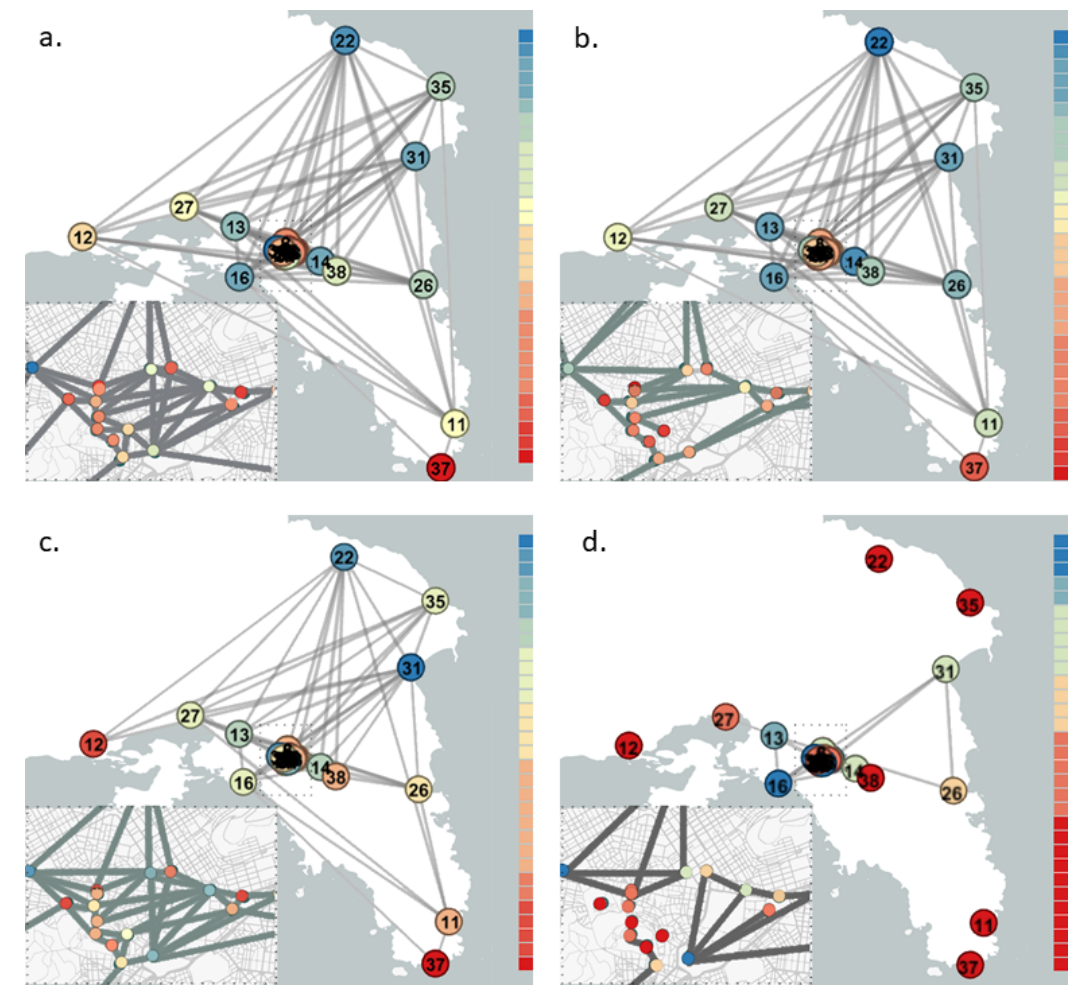

Figure A4. Spatial distribution of degree in the (a) aggregate layer; (b) car layer; (c) pedestrian; and (d) transportation layer (max values are shown on the top and min at the bottom of each color ramp).

\section{References}

1. United Nations World Tourism Organisation (UNWTO). Tourism and Culture. Available online: https://www.unwto.org/ tourism-and-culture (accessed on 30 November 2020).

2. Darbellay, F.; Stock, M. Tourism as complex interdisciplinary research object. Ann. Tour. Res. 2012, 39, 441-458. [CrossRef]

3. Kalantzi, O.; Tsiotas, D.; Polyzos, S. The contribution of tourism in national economies: Evidence of Greece. EJBSS 2016, 5, $41-64$.

4. Polyzos, S. Regional Development, 2nd ed.; Kritiki: Athens, Greece, 2019; ISBN 9789602187302.

5. Polyzos, S.; Tsiotas, D. The contribution of transport infrastructures to the economic and regional development. Theor. Empir. Res. Urban Manag. 2020, 15, 5-23.

6. Csapo, J. The role and importance of cultural tourism in modern tourism industry. Strateg. Tour. Ind.-Micro Macro Perspect. 2012, 10, 201-212.

7. Polyzos, S.; Arabatzis, G.; Tsiantikoudis, S. The attractiveness of archaeological sites in Greece: A spatial analysis. Int. J. Tour. Policy 2007, 1, 246-266. [CrossRef]

8. Rodrigue, J.P.; Comtois, C.; Slack, B. The Geography of Transport Systems; Routledge: New York, NY, USA, 2013.

9. United Nations World Tourism Organisation (UNWTO). Tourism Highlights. 2018. Available online: https://www.e-unwto.org/ doi/pdf/10.18111/9789284419876 (accessed on 30 November 2020).

10. Richards, G. Cultural tourism: A review of recent research and trends. J. Hosp. Tour. Manag. 2018, 36, 12-21. [CrossRef]

11. Wu, M.; Gao, X.; Cao, M.; Papa, E.; Zhu, X. The changes of intergovernmental collaboration dynamic in post-disaster destination management: Network analysis. J. Hosp. Tour. Manag. 2021, 48, 32-45. [CrossRef]

12. Presenza, A.; Cipollina, M. Analysing tourism stakeholders networks. Tour. Rev. 2010, 65, 17-30. [CrossRef]

13. Tran, M.T.; Jeeva, A.S.; Pourabedin, Z. Social network analysis in tourism services distribution channels. Tour. Manag. Perspect. 2016, 18, 59-67. [CrossRef]

14. Shih, H.Y. Network characteristics of drive tourism destinations: An application of network analysis in tourism. Tour. Manag. 2006, 27, 1029-1039. [CrossRef]

15. Kang, S.; Lee, G.; Kim, J.; Park, D. Identifying the spatial structure of the tourist attraction system in South Korea using GIS and network analysis: An application of anchor-point theory. J. Destin. Mark. Manag. 2018, 9, 358-370. [CrossRef]

16. Yang, Y. Understanding tourist attraction cooperation: An application of network analysis to the case of Shanghai, China. J. Destin. Mark. Manag. 2018, 8, 396-411. [CrossRef]

17. Liu, B.; Huang, S.S.; Fu, H. An application of network analysis on tourist attractions: The case of Xinjiang, China. Tour. Manag. 2017, 58, 132-141. [CrossRef] 
18. Li, W.; Guan, H.; Han, Y.; Zhu, H.; Zhao, P. Accessibility of Multimode Transport Facilities to Suburban Tourist Attractions: Analysis Based on Meso or Microcommunity Scale in Beijing. J. Urban Plan. Dev. 2021, 147, 04021026. [CrossRef]

19. Sánchez-Martín, J.M.; Gurría-Gascón, J.L.; García-Berzosa, M.J. The cultural heritage and the shaping of tourist itineraries in rural areas: The case of historical ensembles of Extremadura, Spain. ISPRS Int. J. Geo-Inf. 2020, 9, 200. [CrossRef]

20. Guedes, A.S.; Jiménez, M.I.M. Spatial patterns of cultural tourism in Portugal. Tour. Manag. Perspect. 2015, 16, 107-115. [CrossRef]

21. Lozano, S.; Gutiérrez, E. A complex network analysis of global tourism flows. Int. J. Tour. Res. 2018, 20, 588-604. [CrossRef]

22. Boukas, N.; Ziakas, V.; Boustras, G. Towards reviving post-Olympic Athens as a cultural destination. Curr. Issues Tour. 2012, 15, 89-105. [CrossRef]

23. Gkritzali, A.; Gritzalis, D.; Stavrou, V. Is Xenios Zeus still alive? Destination image of Athens in the years of recession. J. Travel Res. 2018, 57, 540-554. [CrossRef]

24. Papatheodorou, A.; Arvanitis, P. Effect of economic crisis on a prefecture level. Région Et Développement 2014, 39, $183-203$.

25. Economou, A.; Mitoula, R. Tourism Development and Impact on the Environment in the Region of Attica. In Proceedings of the 7th International Conference of Law, Business, Marketing and Corporate Social Responsibilities, London, UK, 4-6 December 2017.

26. Cheirchanteri, G. Industrial Cultural Heritage as an Alternative Tourism Model: Case study of the Wider Lavreotiki area in Southeastern Attica. In IOP Conference Series: Materials Science and Engineering; IOP Publishing: Bristol, UK, 2019; Volume 603, p. 022001.

27. Barabasi, A.-L. Network science. Phil. Trans. R. Soc. A 2013, 371, 20120375. [CrossRef] [PubMed]

28. Barthélemy, M. Spatial networks. Phys. Rep. 2011, 499, 1-101. [CrossRef]

29. Baggio, R.; Scott, N.; Cooper, C. Network science: A review focused on tourism. Ann. Tour. Res. 2010, 37, 802-827. [CrossRef]

30. Tsiotas, D.; Polyzos, S. The complexity in the study of spatial networks: An epistemological approach. Netw. Spat. Econ. 2018 18, 1-32. [CrossRef]

31. Hellenic Statistical Authority-ELSTAT. Museums and Archaeological Sites (Visitors, Receipts). Available online: https://www. statistics.gr/en/statistics/- / publication/SCI21/2019-M12 (accessed on 15 May 2021).

32. Koschutzki, D.; Lehmann, K.; Peeters, L.; Richter, S. Centrality indices. In Network Analysis; Brandes, U., Erlebach, T., Eds.; Springer Publications: Berlin, Germany, 2005; pp. 16-61.

33. Newman, M.E.J. Networks: An Introduction; Oxford University Press: Oxford, UK, 2010.

34. Fortunato, S. Community detection in graphs. Phys. Rep. 2010, 486, 75-174. [CrossRef]

35. Kivela, M.; Arenas, A.; Barthelemy, M.; Gleeson, J.; Moreno, Y.; Porter, M.A. Multilayer networks. J. Complex Netw. 2014, 2, 203-271. [CrossRef]

36. Google Maps. Google Mapping Services. Available online: www.google.gr/maps?hl=el (accessed on 15 April 2021).

37. Tsiotas, D. Detecting different topologies immanent in scale-free networks with the same degree distribution. Proc. Natl. Acad. Sci. USA 2019, 116, 6701-6706. [CrossRef]

38. Boccaletti, S.; Bianconi, G.; Criado, R.; del Genio, C.I.; Gomez-Gardenes, J.; Romance, M.; Sendina-Nadal, I.; Wang, Z.; Zanin, M. The structure and dynamics of multilayer networks. Phys. Rep. 2014, 544, 1-122. [CrossRef]

39. Walpole, R.E.; Myers, R.H.; Myers, S.L.; Ye, K. Probability E Statistics for Engineers E Scientists, 9th ed.; Prentice Hall Publications: New York, NY, USA, 2012.

40. Norusis, M. SPSS 16.0 Advanced Statistical Procedures Companion; Prentice Hall Press: New Jersey, NY, USA, 2008.

41. Albert, R.; Barabasi, A.-L. Statistical mechanics of complex networks. Rev. Mod. Phys. 2002, 74, 1-47. [CrossRef]

42. Tsiotas, D. Detecting differences in the topology of scale-free networks grown under time-dynamic topological fitness. Sci. Rep. 2020, 10, 10630. [CrossRef]

43. Blondel, V.; Guillaume, J.-L.; Lambiotte, R.; Lefebvre, E. Fast unfolding of communities in large networks. J. Stat. Mech. 2008, 10, P10008. [CrossRef]

44. Bastian, M.; Heymann, S.; Jacomy, M. Gephi: An open source software for exploring and manipulating networks. In Proceedings of the Third International ICWSM Conference, San Jose, CA, USA, 17-20 May 2009; The AAAI Press: Menlo Park, CA, USA, 2009; pp. 361-362.

45. TripAdvisor. Hotels in Attica. Available online: https://www.tripadvisor.com.gr/Hotels-g189400-Athens_Attica-Hotels.html (accessed on 25 May 2020).

46. Tsiotas, D. Drawing indicators of economic performance from network topology: The case of the interregional road transportation in Greece. Res. Transp. Econ. 2021, 90, 101004. [CrossRef]

47. Polyzos, S.; Tsiotas, D.; Kantlis, A. Determining the Tourism Developmental Dynamics of the Greek Regions, by using TALC Theory. TOURISMOS Int. Multidiscip. J. Tour. 2013, 8, 159-178.

48. Almeida, J.; Costa, C.; da Silva, F.N. A framework for conflict analysis in spatial planning for tourism. Tour. Manag. Perspect. 2017, 24, 94-106. [CrossRef]

49. Buhalis, D. Tourism in Greece: Strategic analysis and challenges. Curr. Issues Tour. 2001, 4, 440-480. [CrossRef]

50. Karpodini-Dimitriadi, E. Developing cultural tourism in Greece. In Tourism and Cultural Conflicts; CAB International Publishing: Wallingford, UK, 1999; pp. 113-127.

51. Richards, G. Cultural tourism. In Routledge Handbook of Leisure Studies; Routledge: New York, NY, USA, 2013 ; pp. 505-514.

52. Ioannides, D. Strengthening the ties between tourism and economic geography: A theoretical agenda. Prof. Geogr. 1995, 47, 49-60. [CrossRef] 